\title{
A glycopolymer improves vascoelasticity and mucociliary transport of abnormal cystic fibrosis mucus
}

\author{
Courtney M. Fernandez-Petty, ${ }^{1}$ Gareth W. Hughes, ${ }^{2}$ Hannah L. Bowers, ${ }^{1}$ John D. Watson, ${ }^{1}$ \\ Bradley H. Rosen, ${ }^{3,4}$ Stacy M. Townsend, ${ }^{5}$ Carlo Santos, ${ }^{5}$ Caroline E. Ridley, ${ }^{2}$ Kengyeh K. Chu, ${ }^{6,7,8,9,10}$ \\ Susan E. Birket, ${ }^{1,11}$ Yao Li, ${ }^{1,11}$ Hui Min Leung, ${ }^{6,7,8,9,10}$ Marina Mazur, ${ }^{11}$ Bryan A. Garcia, ${ }^{1,11}$ \\ T. Idil Apak Evans, ${ }^{3}$ Emily Falk Libby, ${ }^{11}$ Heather Hathorne, ${ }^{11,12}$ Justin Hanes, ${ }^{13}$ Guillermo J. \\ Tearney, ${ }^{6,7,8,9,10}$ John P. Clancy, ${ }^{14}$ John F. Engelhardt, ${ }^{3,4}$ William E. Swords,, ${ }^{1,11}$ David J. Thornton, ${ }^{2}$ \\ William P. Wiesmann, ${ }^{5}$ Shenda M. Baker, ${ }^{5}$ and Steven M. Rowe ${ }^{1,11,12,15}$ \\ 'Department of Medicine, University of Alabama at Birmingham (UAB), Birmingham, Alabama, USA. ${ }^{2}$ Wellcome Trust \\ Centre for Cell-Matrix Research, School of Biological Sciences, Faculty of Biology, Medicine and Health, University of \\ Manchester, Manchester Academic Health Science Centre, United Kingdom. ${ }^{3}$ Department of Anatomy \& Cell Biology and \\ ${ }^{4}$ Department of Medicine, University of lowa, lowa City, lowa, USA. ${ }^{5}$ Synedgen Inc., Claremont, California, USA. ${ }^{6}$ Wellman \\ Center for Photomedicine, Massachusetts General Hospital, Boston, Massachusetts, USA. Department of Dermatology, \\ Massachusetts General Hospital, Boston, Massachusetts, USA. ${ }^{8} \mathrm{Harvard}$ Medical School, Boston, Massachusetts, USA. \\ ${ }^{9}$ Harvard-MIT Division of Health Sciences and Technology, Cambridge, Massachusetts, USA. ${ }^{10}$ Department of Pathology, \\ Massachusetts General Hospital, Boston, Massachusetts, USA. "'Gregory Fleming James Cystic Fibrosis Research \\ Center, and ${ }^{12}$ Department of Pediatrics, UAB, Birmingham, Alabama, USA. ${ }^{13}$ Center for Nanomedicine and Departments \\ of Biomedical Engineering, Chemical \& Biomolecular Engineering, Environmental Health Sciences, Neurosurgery, \\ Oncology, Ophthalmology, and Pharmacology \& Molecular Sciences, Johns Hopkins University, Baltimore, Maryland, USA \\ ${ }^{14}$ Department of Pediatrics, Cincinnati Children's Hospital Medical Center, Cincinnati, Ohio, USA. ${ }^{15}$ Department of Cell \\ Developmental \& Integrative Biology, UAB, Birmingham, Alabama, USA.
}

Cystic fibrosis (CF) is characterized by increased mucus viscosity and delayed mucociliary clearance that contributes to progressive decline of lung function. Mucus in the respiratory and $\mathrm{Cl}$ tract is excessively adhesive in the presence of airway dehydration and excess extracellular $\mathrm{Ca}^{2+}$ upon mucin release, promoting hyperviscous, densely packed mucins characteristic of CF. Therapies that target mucins directly through ionic interactions remain unexploited. Here we show that poly (acetyl, arginyl) glucosamine (PAAC), a polycationic biopolymer suitable for human use, interacts directly with mucins in a $\mathrm{Ca}^{2+}$-sensitive manner to reduce $\mathrm{CF}$ mucus viscoelasticity and improve its transport. Notably, PAAC induced a linear structure of purified MUC5B and altered its sedimentation profile and viscosity, indicative of proper mucin expansion. In vivo, PAAG nebulization improved mucociliary transport in CF rats with delayed mucus clearance, and cleared mucus plugging in CF ferrets. This study demonstrates the potential use of a synthetic glycopolymer PAAG as a molecular agent that could benefit patients with a broad array of mucus diseases.

Conflict of interest: SMT, WPW, and SMB have an ownership interest Synedgen Inc., which is developing PAAG as proprietary therapeutic.

Copyright: () 2019 American Society for Clinical Investigation

Submitted: November 1, 2018 Accepted: February 28, 2019 Published: April 18, 2019

Reference information: /CI Insight. 2019;4(8):e125954. https://doi. org/10.1172/jii.insight.125954.

\section{Introduction}

Cystic fibrosis (CF) is a genetic disease that causes thick, adherent mucus to accumulate in the lungs, sinuses, digestive tract, and pancreas (1). CF mucus obstructs the airways and causes progressive life-threatening lung infections, and similar processes also occur in a variety of other airway diseases characterized by mucus stasis and airways obstruction. Mucins, the chief structural component of mucus, are negatively charged linear, polymeric glycoproteins that function to protect the epithelial surface of human airways. Aberrantly cohesive and adhesive mucins contribute to mucus hyperviscosity and chronic infection, hallmarks of diseases of mucus stasis (2-4).

Mucins are highly sulfated, polyanionic polysaccharides on a protein backbone that are held together principally by $\mathrm{Ca}^{2+}$ as a positive counterion, which in excess does not allow normal mucin expansion from goblet cells. 
Electrostatic-driven abnormalities that influence mucin conformation are fundamental in mediating the pathophysiology of mucus. Extrusion of mucus, of which mucins are the chief structural component, from glands and goblet cells represents a crucial event in which displacement of $\mathrm{Ca}^{2+}$ from the tightly packed mucin granules must occur to allow appropriate molecular expansion to avoid mucostasis (5-7). In the absence of an environment rich in bicarbonate and sodium to remove and displace $\mathrm{Ca}^{2+}$, as occurs in the event of dysfunctional $\mathrm{CF}$ transmembrane regulator-mediated (CFTR-mediated) ion transport in CF and other lung diseases, mucins are unable to undergo normal posttranslational processing and expansion to their linear form, instead remaining compact, contributing to mucus that is hyperviscous, cohesive, and adhesive to the surface epithelium (7-9). $\mathrm{Ca}^{2+}$ chelation has been investigated as an intervention to change the local ionic environment of airway mucus but has not yet been successful $(7,10-13)$. In contrast, we postulated that a polycationic glycopolymer could modulate the mucin-mucin interactions mediated by $\mathrm{Ca}^{2+}$ to create a solution phase of mucus with different adhesive and viscoelastic properties. The current effort reflects an attempt to directly target the anions present on mucins, altering the intramolecular ionic interactions to displace $\mathrm{Ca}^{2+}$ as a mucin counterion. Unlike chelation, this therapeutic approach may have inherent advantages by not diminishing the free $\mathrm{Ca}^{2+}$ concentrations in the airway and thus not interfering with the cell signaling properties of free $\mathrm{Ca}^{2+}$.

We hypothesized that poly (acetyl, arginyl) glucosamine (PAAG) - a high-MW polyglucosamine that has been chemically modified with arginine moieties to provide a highly positively charged, soluble polymer (Supplemental Figure 1; supplemental material available online with this article; https://doi.org/10.1172/ jci.insight.125954DS1) - would interact preferentially with the mucin sulfates. Because of the high MW of the glycopolymer and because arginine exhibits unique interactions with sulfates (14), these transient intermolecular interactions are expected to displace $\mathrm{Ca}^{2+}$ to affect mucin morphology. In preclinical toxicology studies performed in two species and successfully completed phase I studies in humans, PAAG was non-toxic, safe, and well tolerated. Using CF as a disease model of the airways and gastrointestinal tract, here we show that PAAG interacts with mucins in a $\mathrm{Ca}^{2+}$-dependent manner to reduce mucus viscoelasticity and improve mucus transport, ameliorating mucus stasis in 3 different CF animal models.

\section{Results}

PAAG improves viscoelasticity of CF sputum. The effects of PAAG on the elastic modulus $\left(\mathrm{G}^{\prime}\right)$ and dynamic viscosity $(\eta)$ of $C F$ sputum were assessed by oscillatory rheometry to measure viscoelasticity at extremely low shear (15), to minimize disrupting innate mucus structure. The demographics of these patients are shown in Supplemental Table 1. Sputum samples were aliquoted and incubated at $37^{\circ} \mathrm{C}$ for 2 hours with various concentrations of PAAG or DTT $(100 \mu \mathrm{g} / \mathrm{ml})$, a known mucolytic that acts by reducing disulfide bonds and that served as a positive control, albeit by a distinct mechanism (16). After evaluating a dose response curve, the most effective concentration was determined (Supplemental Figure 2A). The original dose response was performed in induced sputum, which can affect baseline viscoelastic properties; therefore, for subsequent experiments to obtain clinically relevant material, we collected fresh, spontaneously expectorated sputum from patients with CF who were hospitalized for pulmonary exacerbations. We evaluated PAAG $(100-500 \mu \mathrm{g} / \mathrm{ml})$ and observed a significant reduction in dynamic viscosity of CF sputum at low shear rates at $100 \mu \mathrm{g} / \mathrm{ml}$ (Figure 1A; left side of the curve, prior to shear thinning at high shear rates). Similar beneficial effects on the elastic modulus were also apparent after treatment with $100 \mu \mathrm{g} / \mathrm{ml}$ PAAG (Figure 1B). Effective viscosities measured at a shear rate of $0.8 \mathrm{~s}^{-1}$ indicated that the treatment effect was particularly prominent in CF sputum samples that exhibited high dynamic viscosity at baseline (359 $\pm 561 \mathrm{~Pa} \bullet$ s for sputum treated with PBS compared with $62 \pm 97 \mathrm{~Pa} \bullet \mathrm{s}$ for sputum treated with PAAG; Figure 1C). PAAG treatment also significantly reduced elastic modulus across various sputum donors (18.39 \pm 8.72 Pa for PBS vs. $3.02 \pm 1.12 \mathrm{~Pa}$ for PAAG; Figure 1D), with the greatest effect observed in those with the highest elasticity at baseline. As an additional control, sputum with PAAG treatment was compared with treatment with an uncharged, soluble polysaccharide of similar molecular size (polyglucose, hydroxypropyl methyl cellulose [HPMC]), the available mucolytic recombinant human deoxyribonuclease I (DNase; $100 \mu \mathrm{g} / \mathrm{ml}$ ), and the widely used $\mathrm{Ca}^{2+}$-chelating agent EGTA (10 mM). Unlike PAAG, DNase alters the physical properties of mucus by degrading free DNA, a component in mucus largely derived from polymorphonuclear cells (17). Neither PBS nor HPMC lowered the dynamic viscosity of CF sputum measured at a shear rate of $0.8 \mathrm{~s}^{-1}$, whereas PAAG, DNase, DTT, and EGTA significantly lowered dynamic viscosity (Figure 1E). PAAG also reduced viscosity as measured by a change in viscous (loss) modulus $\left(G^{\prime \prime}\right)$ over a frequency sweep (Supplemental Figure 2B). 
G" effects were compared at $0.8 \mathrm{~Hz}$ (Supplemental Figure 2C); again, the most prominent effects in sputa were observed in specimens with the highest viscosity at baseline. The effects of PAAG were independent of changes in solid content of sputum samples, a measure of hydration (Supplemental Figure 2D), and PAAG had no effects on the viscosity of pure lambda DNA (data not shown). These ex vivo results showed that PAAG treatment produced a significant reduction in CF sputum viscosity and elasticity, approaching levels in sputum obtained from normal volunteers.

$P A A G$ reduces viscosity of CF human bronchial epithelial mucus. Since negatively charged glycosaminoglycans are highly expressed on the epithelial surface (18), we hypothesized that PAAG would be particularly beneficial in improving mucus penetrability as measured by effective viscosity on the surface of epithelial cells in situ, where deleterious mucin binding is favored in CF due to the relative deficiency of bicarbonate (7). Primary human bronchial epithelial (HBE) cells enable assessment of airway surface microenvironment characteristics, including ion transport, ciliary beating, mucociliary transport (MCT), and mucus viscosity (19-22). Primary HBE monolayers derived from donors with CF (Phe508del homozygous) were grown at air-liquid interface until terminally differentiated and then treated apically with PAAG or PBS control for 24 hours. Concentrations applied were chosen to achieve therapeutic concentrations after in vivo dilution by airway mucus (estimated at $100 \mu \mathrm{g} / \mathrm{ml}, 250 \mu \mathrm{g} / \mathrm{ml}$, and $500 \mu \mathrm{g} / \mathrm{ml}$ based on mean airway surface liquid (ASL) volume of the $0.33 \mathrm{~cm}^{2}$ Transwell filter and expected delivered dose based on animal studies). To monitor viscosity in situ by particle tracking microrheology (PTM), ciliary beating was stopped with benzalkonium chloride ( $0.1 \%)$ for 60 minutes, and $500 \mathrm{~nm}$ polyethylene glycol-coated (PEG-coated) (muco-inert) particles were applied to determine the effective viscosity of innate ASL $(23,24)$. Representative particle tracings (Figure $1 \mathrm{~F}$ ) indicated significantly greater diffusibility, measured in mean squared displacement (MSD), following PAAG treatment $(500 \mu \mathrm{g} / \mathrm{ml})$, compared with PBS (Figure $1 \mathrm{G})$. The effect of PAAG on the Brownian motion of muco-inert particles was dose dependent, with peak efficacy between 250 and $500 \mu \mathrm{g} / \mathrm{ml}$. Particle tracks were then analyzed by Fourier transformation to determine frequency-dependent viscous modulus $(20,23,25)$. PAAG caused a 2-log reduction in effective viscosity across all frequency ranges, indicating a highly beneficial effect in situ that was not fully evident with ex vivo sputum studies (Figure $1 \mathrm{H})$. This reduction was evident at a very low frequency $(597.0 \pm 56.1 \mathrm{cP}$ for PBS control versus $2.84 \pm 0.11 \mathrm{cP}$ PAAG at $1.0 \mathrm{~Hz}$ ), similar to physiological shear caused by breathing (Figure 1I).

Since we saw a strong effect of DNase in CF sputum, we wanted to compare the relative effects of these agents using PTM and tested for additive benefits, since their mechanisms are complementary. CF HBE monolayers were treated with PBS control, PAAG $(250 \mu \mathrm{g} / \mathrm{ml})$, DNAse $(250 \mu \mathrm{g} / \mathrm{ml})$, or a combination of these agents (Figure 1J). Both agents reduced effective viscosity, but in cell culture studies, PAAG exhibited a greater effect than DNAse, whereas the effect of DNAse in sputum was larger (Figure 1E), probably related to the relatively scant amounts of free DNA in HBE culture monolayers (primarily from occasional cell turnover) as compared with DNA content in CF sputum, which is substantially greater. The potential for PAAG to reduce interaction of mucus with the surface epithelium may also have been a factor in augmenting the response to PAAG in an intact system in which mucociliary interactions may have prominent effects, rather than expectorated samples where these forces are not relevant. Regardless, the combination of PAAG and DNase increased particle diffusion, reflecting distinct and complementary mechanisms and suggesting that PAAG could be used in combination with current therapies used in CF care (Supplemental Figure 2E). This combined treatment reduced effective viscosity across all frequency ranges (Supplemental Figure 2F), including low shear conditions (Figure 1J). Since the experiments in Figure 1, I and J, were done under identical experimental conditions, a combined analysis demonstrated that PAAG significantly reduced viscosity by 32 -fold at $250 \mu \mathrm{g} / \mathrm{ml}(15.7 \mathrm{cP}$, respectively) as compared with vehicle control (490.8 cP; $P<0.01)$.

Given that the effects of PAAG in situ were more prominent than in analysis of ex vivo sputum, these studies also raised the possibility that PAAG may interact with the surface epithelium, altering interactions with the mucus layer. Indeed, like mucins, the glycocalyx of the epithelial surface is negatively charged and is observed to impede CF mucus release from goblet cells, affecting its ability to transport across the epithelium $(18,26)$. In this case, PAAG could modulate non-covalent adhesive forces between the epithelial surface and mucins, binding that may be mediated by divalent calcium $(7,27)$. To confirm the interaction of PAAG with mucus at the cell interface, we sought to directly visualize PAAG on the epithelial surface. WT and CF HBE cells were treated with FITC-labeled PAAG, and the ASL layer was subsequently stained with Texas red. Representative images are shown for WT and CF HBE monolayers (Figure 1K) and revealed that PAAG was heterogeneously distributed throughout the mucus layer, particularly in $\mathrm{CF}$, where mucus can accumulate in 
A

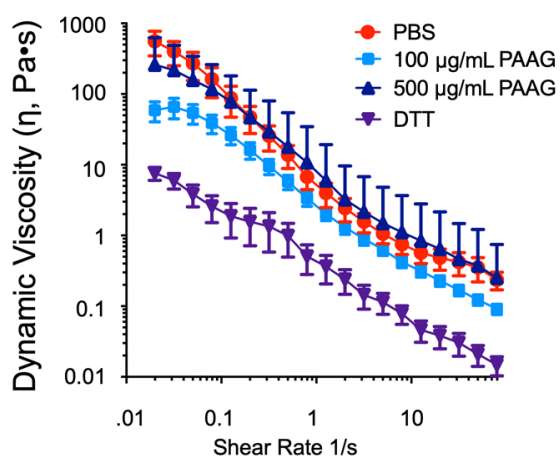

B
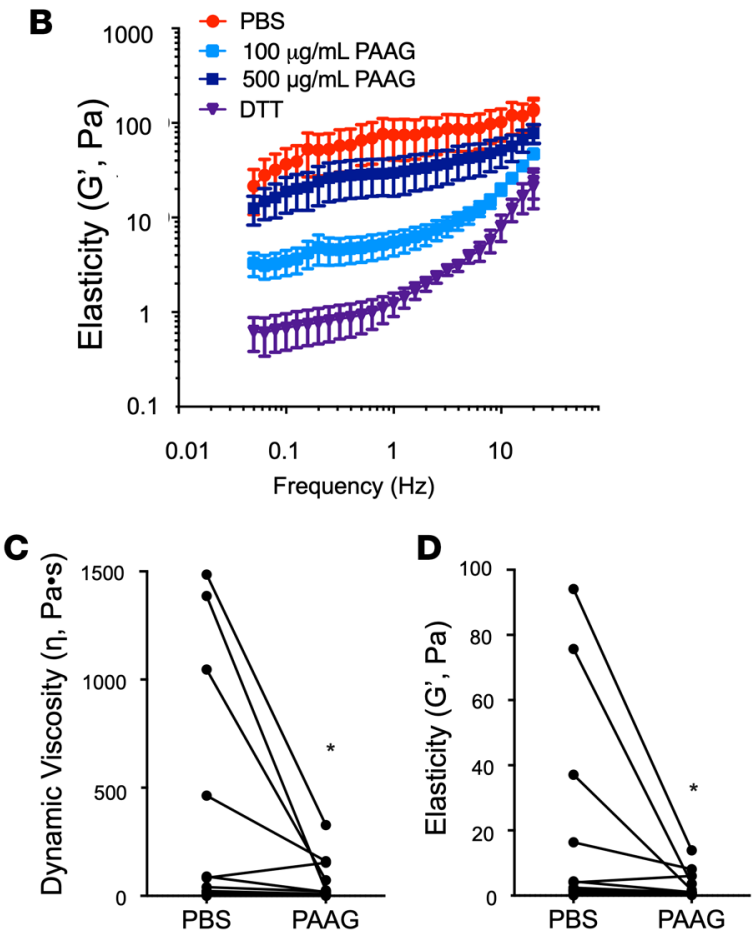

E

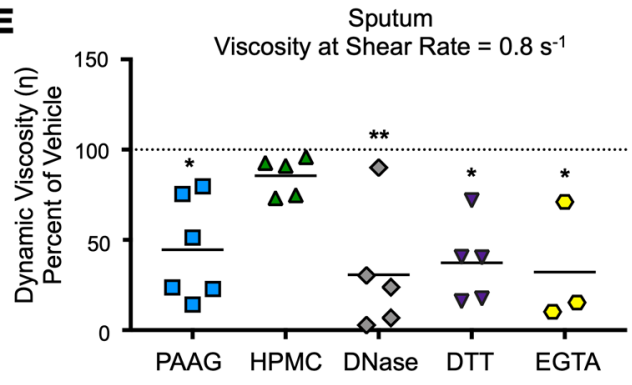

H
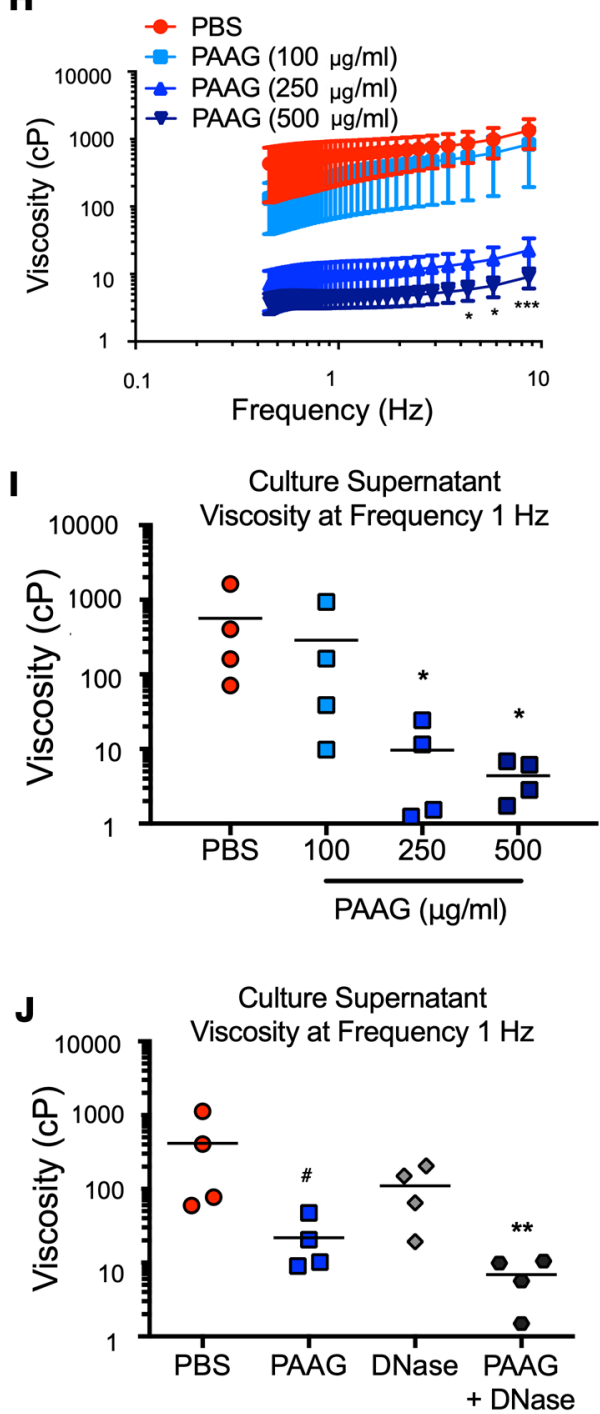

F PAAG $(500 \mu \mathrm{g} / \mathrm{ml})$

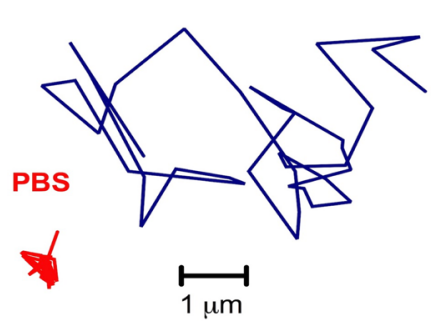

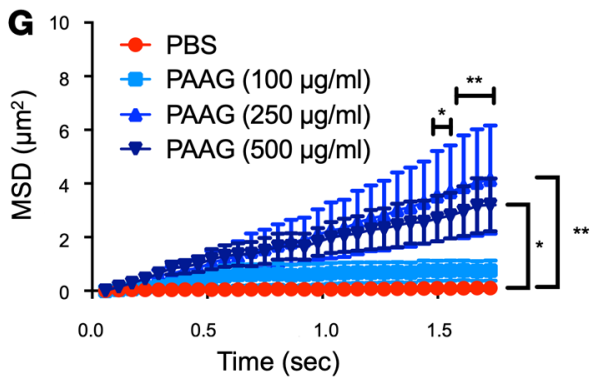

$\mathbf{L}$

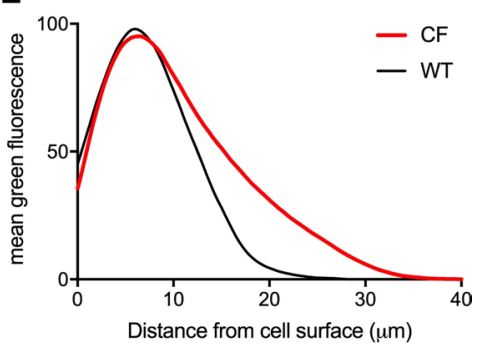


Figure 1. Effect of PAAG on the viscoelastic properties of expectorated CF sputum and mucus from cultured epithelial cells. (A-E) Sputum collected from CF subjects was treated with PBS, PAAG, HPMC, DNase, or DTT as a positive control. (A) Dynamic viscosity ( $\eta$ ) curves show decreased viscosity with PAAG at low (i.e., physiologic) frequency as compared with PBS. (B) Shear-dependent elastic modulus (G') shows an effect of $100 \mu \mathrm{g} / \mathrm{ml}$ PAAG across all frequencies reduction in sputum elasticity compared with control. (C) Summary data of dynamic viscosity ( $\eta$ ) at a frequency sweep of $0.8 \mathrm{~Hz}$ (low but reliable frequency, as expected to occur in vivo) shows a significant decrease in viscosity with $100 \mu \mathrm{g} / \mathrm{ml}$ PAAG treatment. Each line represents a sputum sample; some donors were evaluated more than once on separate clinical encounters $(n=13)$. (D) Similar analysis of sputum elastic modulus $\left(G^{\prime}\right)$. ${ }^{*} P<0.05$ by Wilcoxon's rank test. (E) Summary data of dynamic viscosity $(\eta)$ at a frequency sweep at $0.8 \mathrm{~Hz}$ comparing the effect of PAAC with the addition of the neutral polymer control HPMC, the known mucolytic DNase, and the $\mathrm{Ca}^{2+}$ chelator EGTA. Replicates derived from $n=6$ sputum samples and divided among conditions; ${ }^{*} P<0.05,{ }^{*} P<0.01$ by Kruskal-Wallis test with Dunn's MCT. (F) Representative particle track of 500 nm PEG-coated nanoparticle placed in mucus layer of Phe508del/Phe508del HBE cells treated with PAAG (10 $\mu \mathrm{l}$ of 2, 5, and $10 \mathrm{mg} / \mathrm{ml} \mathrm{PAAG} \mathrm{added} \mathrm{apically,} \mathrm{to} \mathrm{achieve} 100,250$, and $500 \mu \mathrm{g} / \mathrm{ml}$ final concentrations, respectively, in the ASL) versus PBS control 24 hours prior to assay. (C) Summary data plotting mean squared displacement (MSD) versus time. Increased slope is an indicator of increased diffusion and decreased mucus viscosity. Doses shown are estimated ASL concentrations, as described above. (H) Particle tracking microrheology (PTM) analysis of data in $\mathbf{G}(250$ and $500 \mu \mathrm{g} / \mathrm{ml})$ showed a marked decrease in viscosity as compared with vehicle control. Reduced viscosity was seen across all ranges of a frequency sweep $(0.46-17.36 \mathrm{~Hz})$, indicating that the effect was robust. Viscosity of normal HBE cells at $1.0 \mathrm{~Hz}$ is $17.7 \pm 4.3 \mathrm{cP}$ (23). (I) Summary data of frequency sweep at $1 \mathrm{~Hz}$. (J) Summary data at frequency sweep $1 \mathrm{~Hz}$ with PAAG compared with the mucolytic DNase. $n=4$ filters/condition. ${ }^{*} P<0.05$, ${ }^{* *} P<0.01$, ${ }^{* *} P<0.001,{ }^{*} P=0.11$ by 2 -way ANOVA with Tukey's (G and $\mathbf{H}$ ) or by Kruskal-Wallis test with Dunn's MCT (I and J). (K and I) Fluorescence image of WT HBE and CF HBE monolayer treated with $250 \mu \mathrm{g} / \mathrm{ml}$ of PAAG-FITC shows that PAAG intermingles within the mucus layer (K) and is predominantly localized within close proximity to the epithelial surface (L). ASL dyed with Texas red to provide contrast. Scale bar: $10 \mu \mathrm{m} ; n=4$ filters/condition.

some locations of the monolayer. When quantified, PAAG distribution was disproportionately prominent along the epithelial surface and peaked where mucus encounters the surface of the cilia $(\sim 7.2 \mu \mathrm{m}$, the height of an extended cilium; Figure 1L). FITC-labeled PAAG was also observed to have residual presence on the surface of A549 epithelial cells despite repetitive washing (Supplemental Figure 3A); similar findings were obtained in A431 skin epithelial cells (Supplemental Figure 3B). Representative images of A549 epithelial cells from control at 0 hours (Supplemental Figure 3C), ands FITC-PAAG cells at 0 hours (Supplemental Figure 3D) and 5 hours after a single wash (Supplemental Figure 3E) are shown. PAAG adhered to the surface of A549 epithelial cells, even after gentle rinsing hourly. Likely because of its positive charge and affinity for GAGs on the surface of the cells, PAAG adhered as a thin layer, and did not accumulate. These effects were recapitulated in vivo, as FITC-labeled PAAG was bound to the surface epithelium upon nebulization to ferrets (Supplemental Figure 4, A and B). In aggregate, these data indicated that PAAG localizes to the epithelial surface to reduce adhesion between adherent mucins and the epithelial surface.

$P A A G$ increases $C F$ mucus transportability. We hypothesized that reduced mucus viscosity conferred by PAAG at the epithelial surface, an indirect indicator of its adhesiveness when examined in situ (20), would translate into improved MCT. To test this hypothesis, we treated primary CF HBE cell culture monolayers under physiologic conditions $\left(37^{\circ} \mathrm{C}, 5 \% \mathrm{CO}_{2}\right)$ and imaged using one-micron resolution optical coherence tomography $(\mu \mathrm{OCT})$, a real-time imaging modality that visualizes the microanatomy and functional aspects of the airway surface $(24,28)$. As opposed to cells treated with PBS vehicle, PAAG-treated cells exhibited a less compact, more heterogeneous mucus layer (Figure 2, A and B). Quantitative analysis indicated a 57\% increase in MCT rate in cells treated with PAAG $(250 \mu \mathrm{g} / \mathrm{ml})$ compared with the PBS control (Figure 2C), even though there was no effect on ASL depth. Commensurate with these changes, ciliary beat frequency increased with PAAG treatment (Figure 2D). Reduced viscosity and elasticity in CF sputum with PAAG treatment and improved MCT of CF mucus suggest that PAAG may accelerate mucus clearance derived from sputum of CF patients (once expectorated, airway mucus is termed sputum, and is a heterogeneous mixture of mucins, proteins, cells, electrolytes, and extruded DNA). To address whether PAAG improved sputum clearance or transportability, human CF sputum was treated with various concentrations of PAAG or vehicle control for 2 hours, then added to the surface of excised rat trachea and imaged using $\mu$ OCT to visualize transport of the sputum along the tracheal surface. The experimental schematic in Supplemental Figure 5A shows points of imaging and randomized additions of each sample. Representative $\mu \mathrm{OCT}$ images showed that PBS control sputum (Figure 2E and Supplemental Video 1) was heterogenous and poorly transported, whereas PAAG-treated $(250 \mu \mathrm{g} / \mathrm{ml})$ sputum was more rapidly transported in a homogenous fashion (Figure $2 \mathrm{~F}$ and Supplemental Video 2). Time-dependent reprocessed images readily quantify MCT rates of sputum (Figure 2, G and $\mathrm{H}$ ); for consistency, analysis was performed $<50 \mu \mathrm{m}$ above the epithelial surface, since above this height, mucus flow is nonlaminar. MCT rates were significantly faster for sputum samples that were treated with PAAG $(3.91 \pm 1.89 \mathrm{~mm} / \mathrm{min}$ PAAG; Figure $2, \mathrm{H}$ and I; $P<0.01)$ as compared with those treated with PBS $(1.62 \pm 0.56 \mathrm{~mm} / \mathrm{min}$, Figure $2, G$ and I) or HPMC $(0.63 \mathrm{~mm} / \mathrm{min})$. The mucus reductant DTT also had a prominent effect, as expected (Figure 2I). 

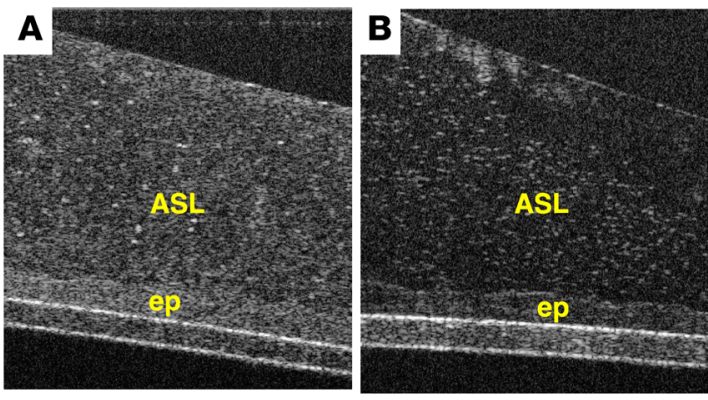

C

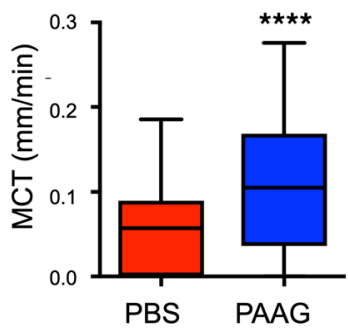

D

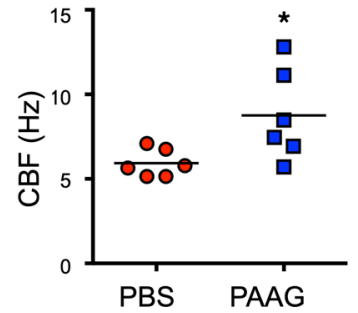

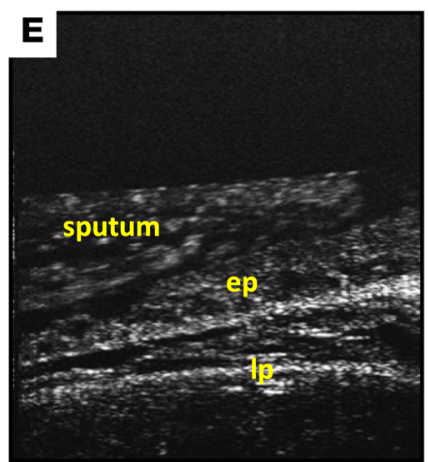
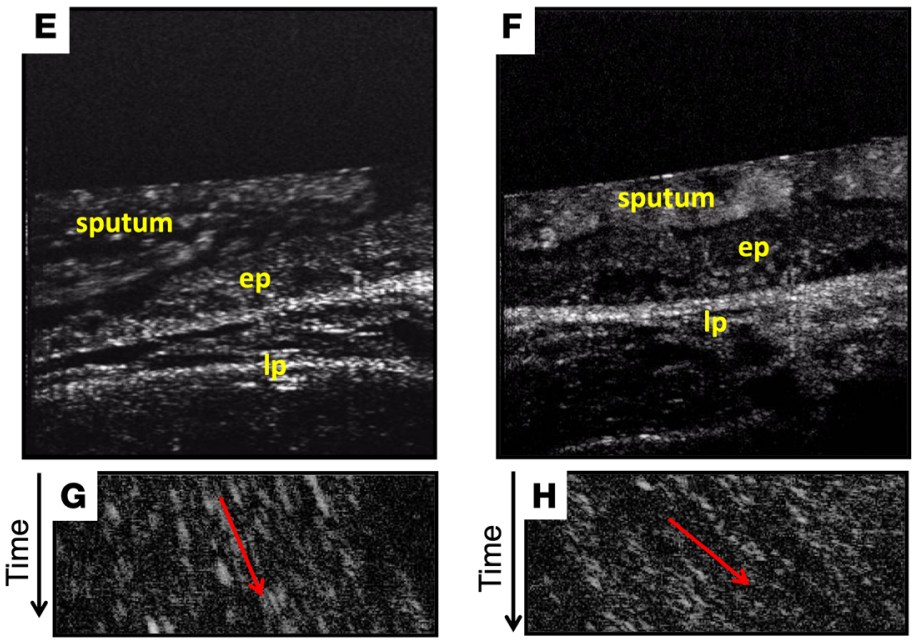

I

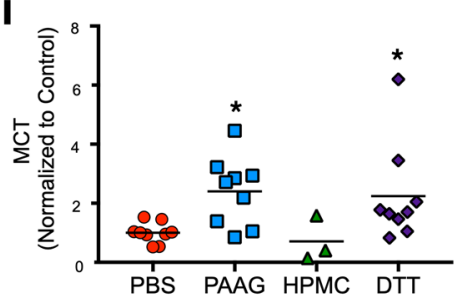

J

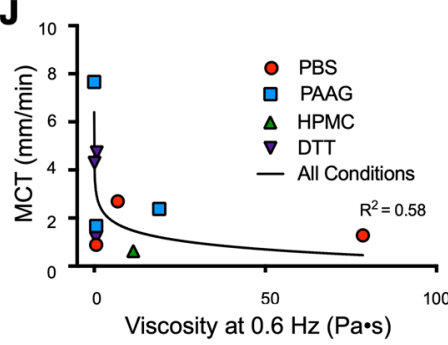

Figure 2. Effect of PAAG on mucus transportability. (A-D) Representative $\mu$ OCT images of CF HBE monolayers treated with PBS control (A) or PAAC (250 $\mu \mathrm{g} / \mathrm{ml}$ airway surface liquid [ASL] concentration, 24 hours) (B) demonstrated clearly reduced reflectivity of the mucus layer following PAAG treatment, indicating reduced viscosity in situ without altering the integrity of the cell monolayer. ep, epithelium. Quantitative data from $\mu$ OCT video imaging/rate imaging showed increased mucociliary transport (MCT; C) and improved ciliary beat frequency (CBF; D) following PAAC treatment. $n=6$ filters/condition (1 CF donor); ${ }^{*} P<0.05,{ }^{* * * *} P<0.0001$ by unpaired $t$ test. (E-J) Expectorated CF sputum was treated with PAAC or HPMC (100 $\left.\mu \mathrm{g} / \mathrm{ml} \times 2 \mathrm{hours}\right)$, or PBS control, and then added to the surface of freshly excised rat trachea while monitoring by $\mu$ OCT. DTT-treated sputum (100 $\mu \mathrm{g} / \mathrm{ml} \times 2 \mathrm{hours})$ was added at the end of each experiment as a control. Representative $\mu$ OCT images from rat trachea after the addition of PBS-treated CF sputum (E) or PAAG-treated CF sputum (F). Ip, lamina propria. Time-dependent reprocessed image showing tracks of sputum particles positioned less than $50 \mu \mathrm{m}$ above the epithelial surface of PBS-treated CF sputum (G) or PAAG-treated CF sputum (H); the more horizontal direction of particle streaks (red arrow) indicates more rapid transport. (I) Summary data showing effect of PAAG on CF sputum transportability. (J) Correlation between MCT and viscosity. $n=3$ sputum donors. ${ }^{*} P<$ 0.05 by Kruskal-Wallis test with Dunn's MCT; $r^{2}=0.58$ for semi-log relationship.

Contemporaneous estimates of sputum dynamic viscosity, which again demonstrated the beneficial effects of PAAG treatment (Supplemental Figure 5, B and C), allowed comparison between sputum viscosity and MCT. A clear exponential relationship was apparent (curve fit $r^{2}=0.58, P<0.05$ ) and demonstrated that sputum samples with the lowest viscosities had the highest MCT rates, and a clear threshold of $\sim 10 \mathrm{cP}$, above which mucus transport was substantially inhibited (Figure $2 \mathrm{~J}$ ). These data also indicate that small changes in viscosity can result in large changes in transport rates, particularly as sputum viscosity approaches this threshold.

$P A A G$ interacts with mucins to alter structure. High concentrations of $\mathrm{Ca}^{2+}$ are present in mucin granules and function to charge-shield negatively charged mucin polymers, allowing them to pack tightly and condense via N-terminal site(s) that bind $\mathrm{Ca}^{2+}(29)$. Upon mucin exocytosis, MUC5B expansion requires bicarbonate to liberate $\mathrm{Ca}^{2+}$ from $\mathrm{N}$-terminal binding sites, allowing for normal posttranslational processing $(5,27,29)$. In the absence of normal epithelial bicarbonate secretion, as observed in CF and possibly other related conditions (30), $\mathrm{Ca}^{2+}$ is not chelated, thus increasing the condensed form of mucin. Consequently, mucus exhibits increased viscosity and adhesion, probably through electrostatic binding mediated by $\mathrm{Ca}^{2+}$ between mucins $(7,27)$ and the glycosaminoglycan-rich epithelial surface $(18,31)$, although covalent bonds such as disulfide bridges may also contribute, particularly as mucins are exposed to the oxidative environment posed by an inflamed airway $(18,32)$. 
Given the effects of PAAG on mucus viscoelasticity, we tested its effects on purified mucins and dependence on extracellular $\mathrm{Ca}^{2+}$ concentration. Purified MUC5B has a network of entangled chains (Figure $3 \mathrm{~A}$ ) that became more compact in the presence of high $\mathrm{Ca}^{2+}$ (Figure 3D) (8). With PAAG treatment, the majority of mucin polymers took on a more linear, extended form in solution (Figure 3B and Supplemental Figure 6E) that migrated at low sucrose concentration by rate-zonal centrifugation (Supplemental Figure $6 \mathrm{~A}$ ) and was more comparable to fully mature forms found in the normal epithelial environment. In addition, a minority of MUC5B molecules ( 25\%) co-sedimented with PAAG (pellet, see Supplemental Figure 6A) and FITC-labeled PAAG (Supplemental Figure 7A), although mucins remained linear on the periphery of the aggregated structures in the pellet (white arrows, Supplemental Figure 6D). Furthermore, the more slowly sedimenting mucins seen at lower sucrose concentrations also co-sedimented with PAAG (Supplemental Figure 7, B-D). In contrast, mucin conformation and sedimentation were unchanged with addition of the neutral soluble polysaccharide HPMC (Figure 3C and Supplemental Figure 6B). As predicted, the conformational changes observed when purified MUC5B was incubated with PAAG were largely abrogated with high $\mathrm{Ca}^{2+}$, evidenced by the presence of globular mucin (Figure 3E, black arrows) and aggregates (Supplemental Figure 6C, right side of curve), although linear forms did occur on the periphery of the molecule (Figure 3E, white arrows; Supplemental Figure 6C, left side of curve).

To confirm that PAAG influenced $\mathrm{Ca}^{2+}$-dependent $\mathrm{N}$-terminal binding sites to alter mucin structure, we evaluated N-terminal mucin multimerization by analytical ultracentrifugation of a preparation of recombinant, dimeric MUC5B N-terminal protein (NT5B dimer). PAAG treatment increased the proportion of NTB dimers (and monomers) and diminished $\mathrm{Ca}^{2+}$-dependent non-covalent assembly of NT5B dimers into multimers (Supplemental Figure 8). These findings indicate that PAAG competes for $\mathrm{Ca}^{2+}$ binding sites in the N-terminus of MUC5B and explain the formation of slowly migrating linear mucin forms observed with PAAG treatment. PAAG had similar effects on sedimentation of MUC5AC in preliminary experiments (data not shown), but additional studies focused on its effects on MUC5B.

Using PTM to estimate the viscosity of purified MUC5B, PAAG increased the Brownian motion of particles, indicated by representative particle tracings (Figure 3F). The MSD of tracked particles was increased (Figure 3G), which corresponded to decreased effective viscosity (Figure 3H), and these results were consistent when extended to 3 seconds (data not shown). In total, these findings indicate that the effects of PAAG on mucus viscosity are correlated with altered structure of mucins and are dependent on $\left[\mathrm{Ca}^{2+}\right]$.

$P A A G$ resolves mucus obstruction in vivo. We sought to test whether the effects of PAAG on mucus viscoelasticity and adhesion could be recapitulated in animal models that produce aberrant $\mathrm{CF}$ mucus. $\mathrm{Cft} \mathrm{r}^{-1}$ mice exhibit distal intestinal mucus obstruction, which typically presents as the animals reach weaning age as mucus and stool obstruct the terminal ileum (33). PAAG treatment by gavage 3 times daily, resulting in negligible osmotic load, was initiated at weaning and significantly improved survival of CF mice (Figure 4A) by preventing distal intestinal obstruction syndrome (DIOS) (Figure 4, C and F). PAAG treatment also improved weight gain in surviving CF mice compared with gavage controls (Figure 4B). Prevention of mucus impaction by PAAG treatment improved mucosal integrity, as indicated by histologic analysis of intestine (Figure 4, D and G). Alcian blue/PAS staining of the colon revealed substantially less mucus impaction with PAAG treatment (Figure 4H) as compared with control (Figure 4E). These results provided in vivo confirmation of the efficacy of PAAG in repairing disordered CF mucus known to occur within the intestine due to absent bicarbonate transport (7).

With improvement in mucus transport in CF cells and tissues, we sought to evaluate the effects of PAAG in vivo using an animal model of $\mathrm{CF}$ that, unlike in mice, exhibits delayed mucus transport in the airways. Using $\mathrm{Cftr}^{-1}$ rats aged to 6 months in which delayed MCT occurs due to abnormally viscous mucus (34), PAAG (250 $\mu \mathrm{g} / \mathrm{ml} \times 20 \mathrm{ml}$ over 45 minutes) or glycerol vehicle control was nebulized once daily for 14 days, as described in the experimental design shown in Supplemental Figure 9. Upon euthanasia, en bloc $\mu$ OCT imaging of the respiratory system was performed on the trachea to measure mucus transport using a method complementary to in vitro studies. Representative $\mu \mathrm{OCT}$ images showed that control-treated animals (Figure 4, I and K, and Supplemental Video 3) had minimal mucus transport, whereas PAAG treatment (Figure 4, J and L, and Supplemental Video 4) accelerated mucus transport 3.5-fold compared with control, achieving approximately 44\% of MCT rates in normal rats (Figure 4M), confirming rates of WT animals in our previous study (34).

$C f t r^{-1}$ ferrets that survive beyond weaning exhibit mucus retention typical of CF lung disease $(35,36)$, resulting in airway obstruction and atelectasis, as revealed by computed tomography. In an adult ferret with a left upper lobe that was completely occluded with mucus (Figure 4N 390 days), mucus obstruction and atelectasis were recalcitrant to copious installation of intrabronchial saline (Figure $4 \mathrm{~N}, 420$ days). In con- 


\section{A}

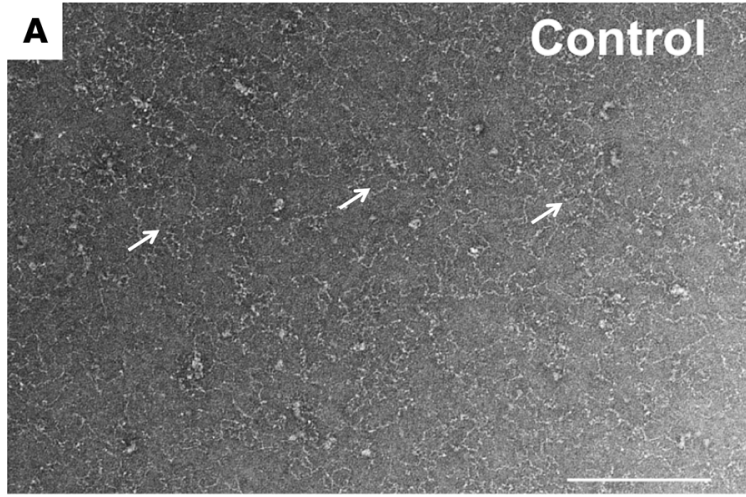

\section{C}

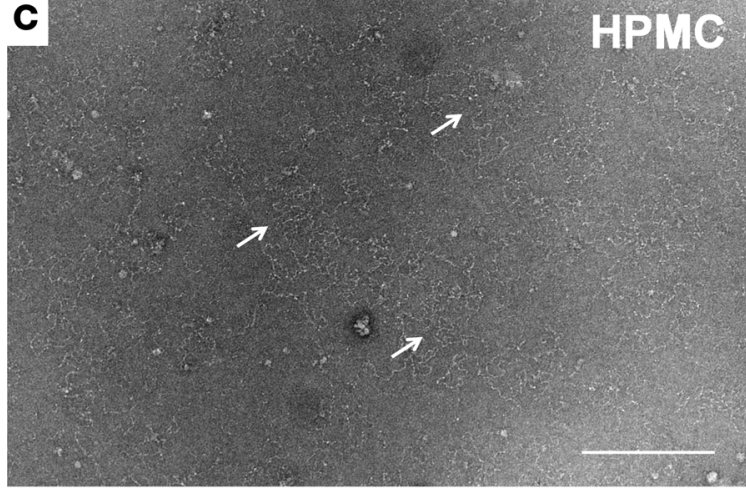

$\mathbf{E}$
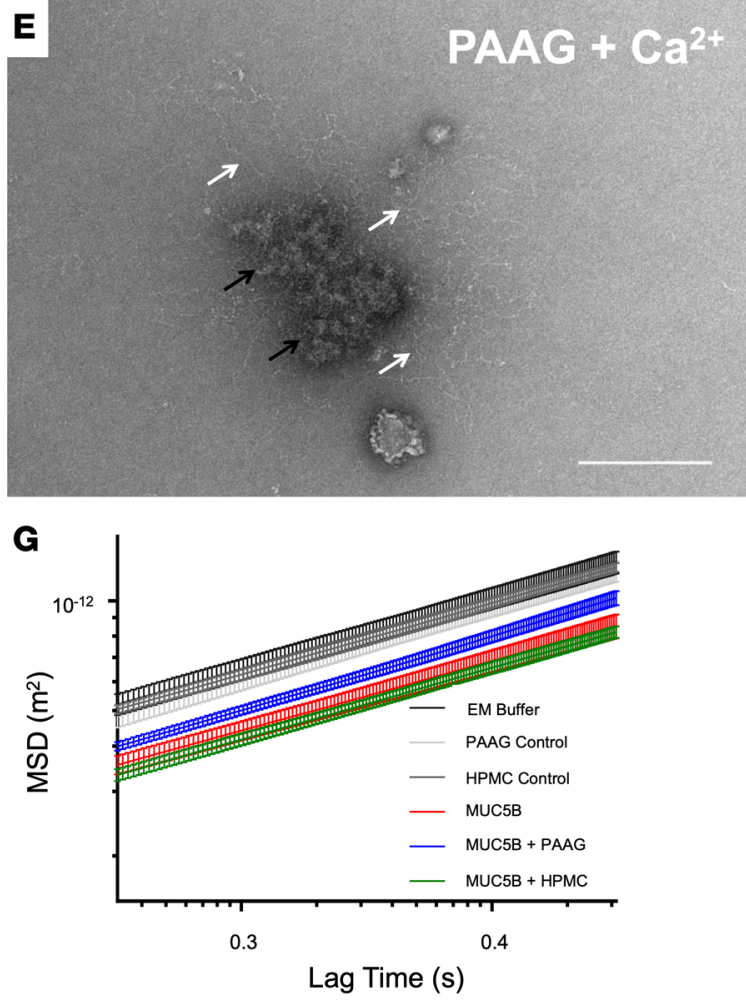
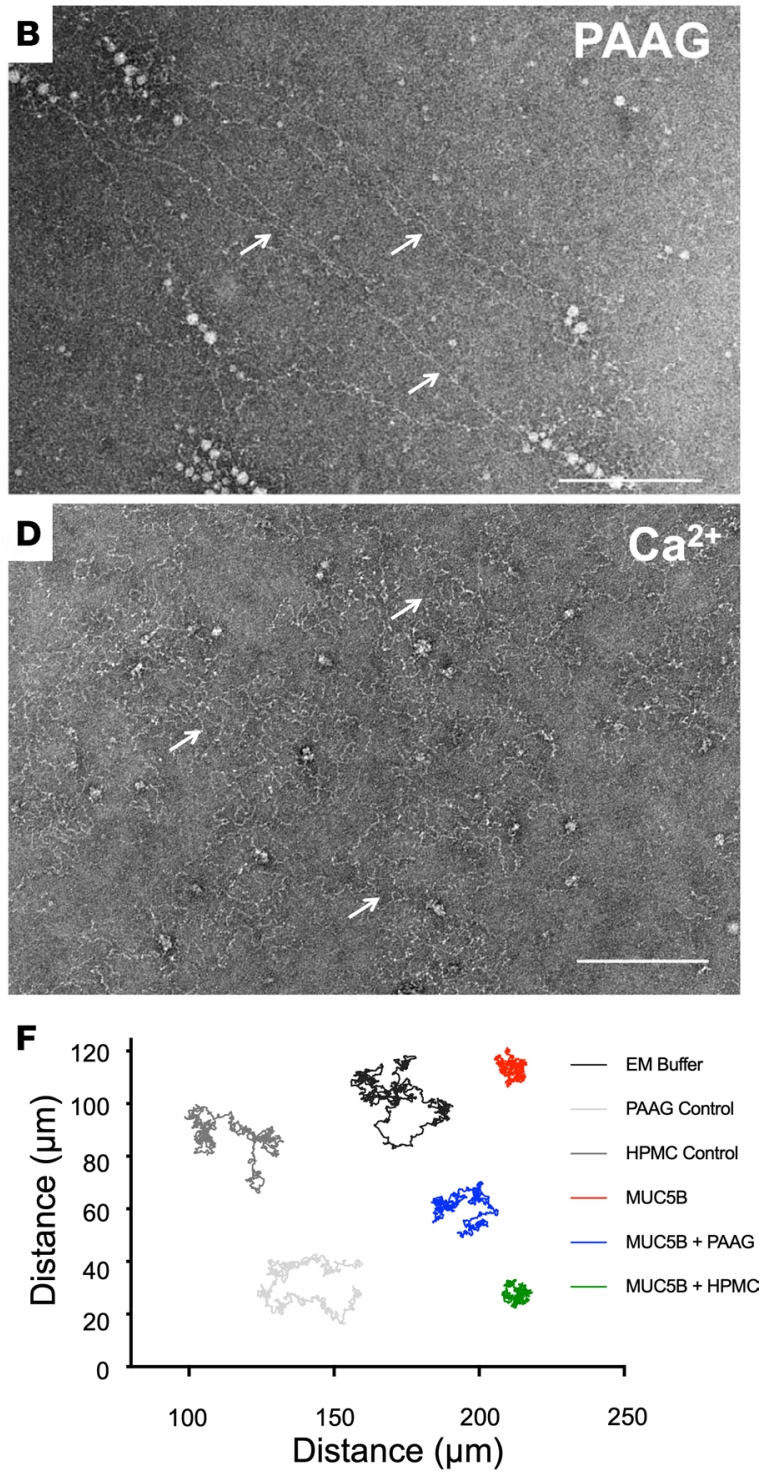

H

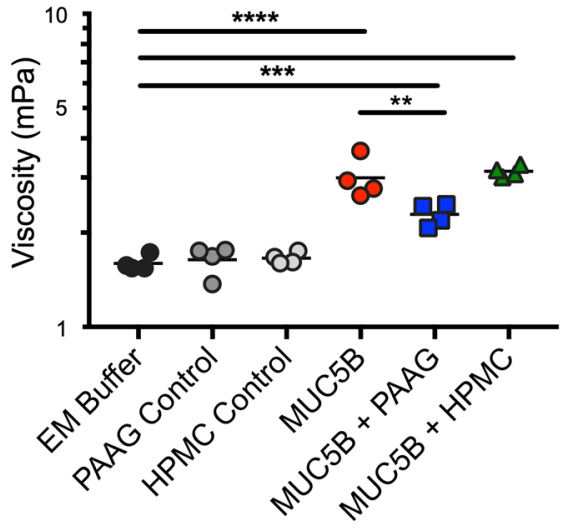

Figure 3. The MUC5B network is structurally altered in the presence of PAAG. Effects of PAAG on purified MUC5B were analyzed using TEM and PTM; scale bars: $200 \mathrm{~nm}$. (A-E) MUC5B at a concentration $50 \mu \mathrm{g} / \mathrm{ml}$ was visualized untreated and in the presence of $100 \mu \mathrm{g} / \mathrm{ml}$ PAAG, $100 \mu \mathrm{g} / \mathrm{ml}$ HPMC, $10 \mu \mathrm{g} / \mathrm{ml} \mathrm{Ca}^{2+}$ individually or in combination. Black and white arrows highlight the position of MUC5B polymers. A linearization of mucin is observed in the presence of PAAG, while HPMC treatment results in no such conformational change. Representative images are shown. (F-H) MSDs of 505-nm polystyrene beads (in $0.1 \mathrm{M} \mathrm{NaCl}, 10 \mathrm{mM}$ Tris- $\mathrm{HCl}$ solutions with $150 \mu \mathrm{g} / \mathrm{ml} \mathrm{MUC5B}, 100 \mu \mathrm{g} / \mathrm{ml} \mathrm{PAAG}, 100 \mu \mathrm{g} / \mathrm{ml} \mathrm{HPMC}$ or in combination) were derived from 15 videos per sample, and repeated on 4 separate occasions. Representative tracks are shown in F. MSD values (G) were then used to derive the viscosity of each sample (H). $n=4$ /condition; ${ }^{* *} P<0.01,{ }^{* *} P<0.01,{ }^{* * * *} P<0.0001$ by 1-way ANOVA with Tukey's. 
A

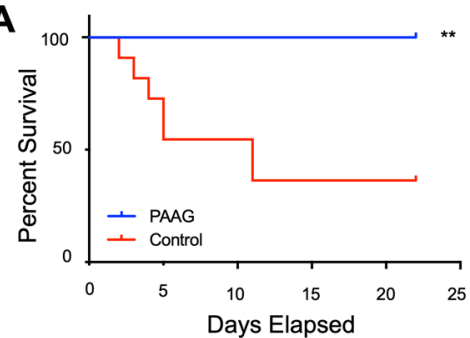

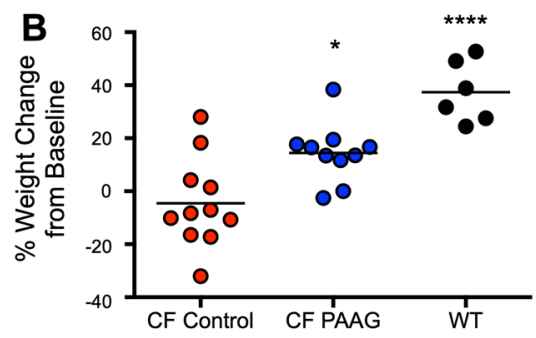
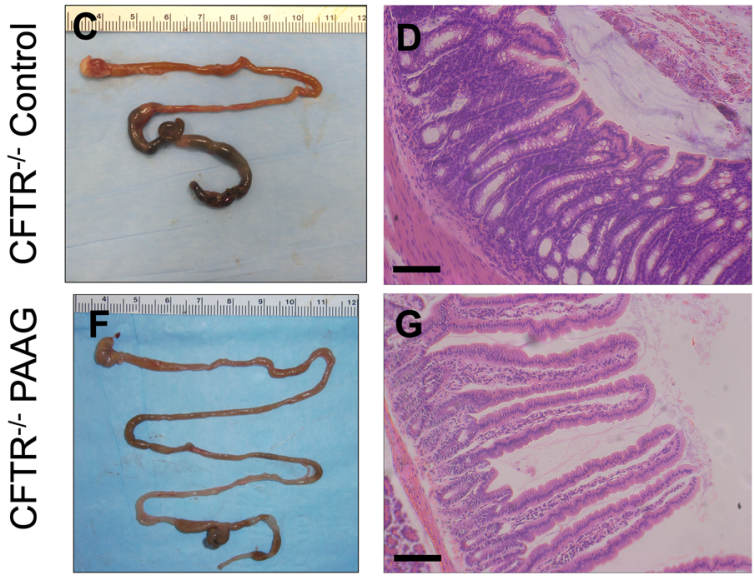
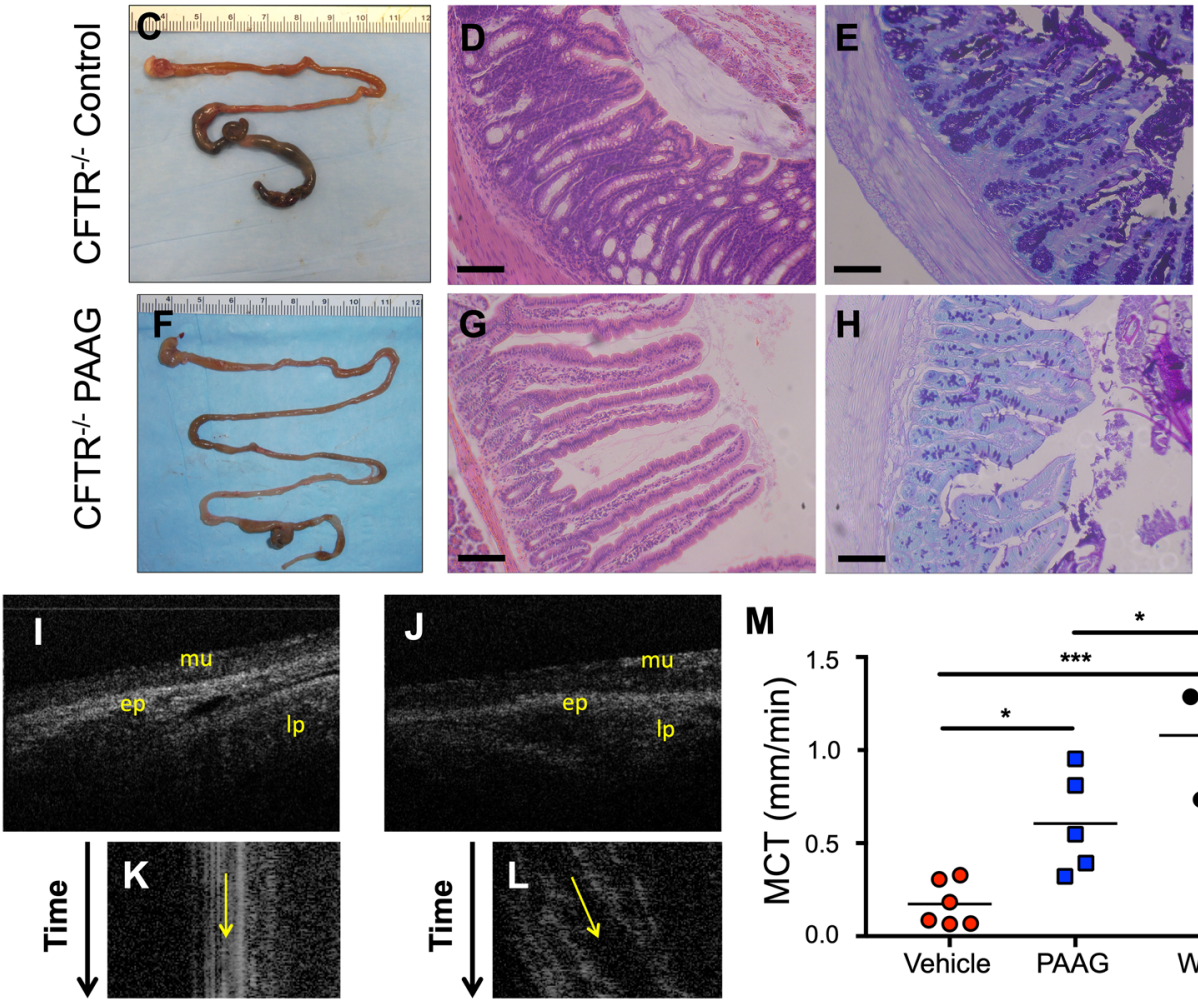

M

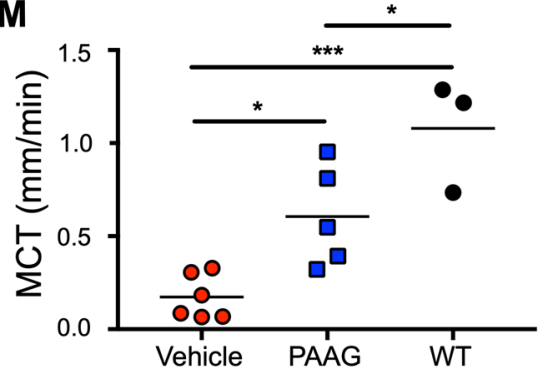

$\mathbf{N}$
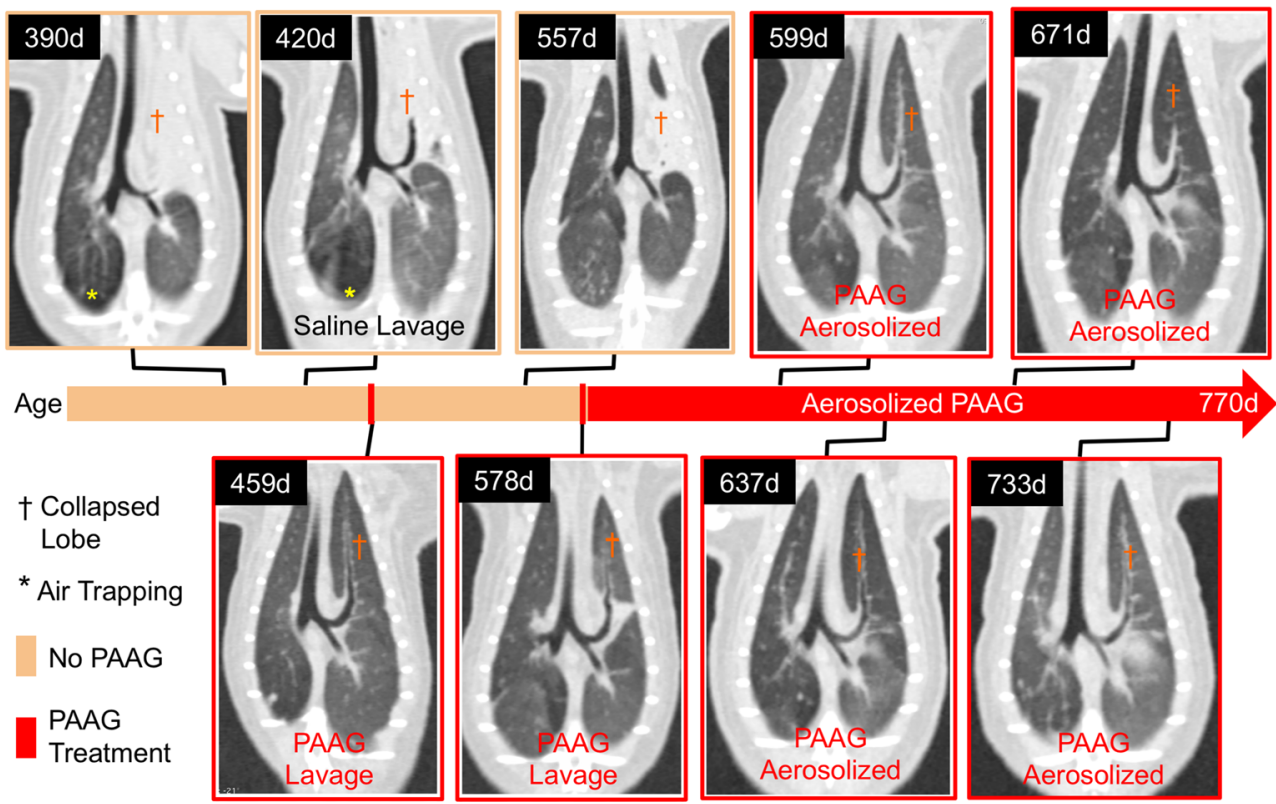

Figure 4. PAAG resolves mucus obstruction in vivo. (A-H) Mice at weaning age were given PAAC (40 $\mathrm{mg} / \mathrm{kg} / \mathrm{d}$ ) by oral gavage divided 3 times daily for 21 days while initiated on a regular diet. (A) Kaplan-Meier survival curves ${ }^{*} P=0.01$. (B) Weight change in mice. Prevention of DIOS as shown by representative images of gross intestine of control (C) and PAAG-treated (F) mice. H\&E staining of control (D) and PAAG-treated (G) intestine. AB/PAS staining of control (E) and PAAG-treated (H) intestine. Scale bars: 100 $\mu \mathrm{m} . n=10$ CF PAAG-treated, 11 CF control-treated, and 6 WT control mice. (I-M) Representative $\mu \mathrm{OCT}$ images from rat tracheas from vehicle-treated animals (I) or PAAG-treated animals (J). Time-dependent reprocessed image showing tracks of mucus particles from vehicle-treated animals (K) or PAAG-treated animals (L). (M) Summary data showing effect of PAAG on MCT rates in $\mathrm{Cfrr}^{--}$rats after 14 days of treatment in comparison to WT littermate controls. (N) Effect of PAAG in a CF ferret. Serial high-resolution CT images demonstrated mucus obstructing the left cranial (upper) lobe $(\dagger)$. Following identification of the obstructed lobe (390 days), bronchoscopic lavage with saline was unsuccessful in removing mucus (420 days). PAAG was instilled by bronchoscopy to the lung segment, and mucus was successfully removed (459 days). Three months after this single use of PAAG, surveillance imaging revealed recurrent mucus obstruction (557 days), which was responsive to directed PAAG lavage (578 days). Following the second lobe recovery, this CF ferret was started on aerosolized PAAC twice weekly, and CT imaging was obtained to follow progress. The lung remained open for the next 192 days (6.4 calendar months) while the ferret was on aerosolized PAAG, without complications or clinical issues. The CF ferret remained on combination antibiotics for the entire lifespan (770 days) until it was euthanized. ${ }^{*} P<$ $0.05,{ }^{* *} P<0.001,{ }^{* * *} P<0.0001$ by 1-way ANOVA with Tukey's. 
trast, intrabronchial PAAG completely cleared the mucus obstruction and reopened the airway, resolving atelectasis (Figure 4N, 459 days). Mucus obstruction recurred 3 months later in the same ferret (Figure $4 \mathrm{~N}$, 557 days). Again, PAAG resolved the recurrent obstruction (Figure 4N, 578 days) and exhibited sustained benefit upon chronic nebulization (Figure 4N, 599, 637, 671, 733 days).

In an exploratory proteomic analysis of bronchoalveolar lavage (BAL) fluid from PAAG-treated ferrets ( $n=3$ ferrets, twice weekly for 8 weeks via inhalation) as compared with pretreatment baseline, we found decreased Muc5b protein expression ( 3 fold, $P<0.0001$ ), and also several indicators of inflammation with statistically significant reductions, including neutrophil elastase (ELANE), calprotectin (S100A12, S100A8, and S100A9), myeloperoxidase (MPO), and matrix metallopeptidase [MMP-8 and MMP-9]), among others (Supplemental Table 2 and Supplemental Data). These findings suggest that PAAG is highly efficacious in complementary models of CF involving completely different organ systems, but with the common mechanism involving mucus obstruction due to aberrant mucus formation.

\section{Discussion}

In the $\mathrm{CF}$ airway, the ionic environment is altered relative to normal conditions, resulting in mucus that is dehydrated, acidic, viscous, and adhesive $(9,24,34,37)$. Treatments to modify the salinity and hydration and of the airway, such as hypertonic saline, have been effective in improving mucus clearance and clinical outcome, but do not normalize mucus clearance $(38,39)$. Agents that target mucus directly, such as drugs intended to improve the oxidative status of mucus or chelate calcium, with the promise of restoring normal mucin confirmation, remain in development and have not yet been shown to be efficacious. Here we demonstrate the efficacy of an approach to directly target the confirmation of mucins with polycationic molecules that displace $\mathrm{Ca}^{2+}$ from the mucins themselves, rather than through a global change in ionic strength or environment.

We show that the biopolymer PAAG normalized CF mucus structure, altering its viscoelastic properties and ability to undergo vectoral motion by the MCT apparatus. A more normal molecular structure of MUC5B resulted from altering inter-domain, non-covalent binding at the $\mathrm{N}$-terminus by interfering with electrostatically mediated relationships within mucin polymers (Figure 3) (40). This improved mucus viscosity, in both purified MUC5B and biologic mucus samples in situ (Figures 1-3). Similar effects were observed in multiple organ systems and across species that resulted in restored MCT in vitro and in CF sputum ex vivo, 2 model systems that strongly predict clinical efficacy (Figure 4) $(22,38)$. These beneficial effects translated to improved MCT and inflammatory status in CFTR-deficient respiratory models and resolved obstruction in the CF GI tract in vivo. One way that MCC could be improved is increasing detachment of mucus from submucosal glands. This has been shown as a mechanism relevant in lung $(41,42)$ and may explain why we see improvement in MCT in the CF rat. While PAAG may also bind and alter the viscosity of free DNA, which is negatively charged, we suspect mucin interactions are the dominant mechanism based on relative concentrations of these molecules in the airway. These results establish preclinical relevance and emphasize the importance of abnormal mucus as a unifying mechanism across various manifestations of CF. The magnitude of improvement, exceeding approximately $50 \%$ of WT mucus transport in normal rats, suggests the potential for clinical benefit. Inhaled PAAG has been found to be safe in normal human volunteers, supporting therapeutic testing among CF patients (our unpublished observations). Of note, the most effective dose of PAAG varied depending on the experimental system, which is likely due to differences in the ratio of mucin (or other interacting glycopolymers) and PAAG, depending on the model used. This will be important when defining the optimal dose in patients as determined by dose-ranging studies.

Increased mucus viscosity is a major contributor to dysfunctional mucus clearance in CF and in other respiratory diseases, representing a potential therapeutic target that has not been fully exploited. PAAG normalized the structure of purified MUC5B (Figure 3) and its sedimentation pattern (Supplemental Figures 5 and 6), features hypothesized to associate with its viscoelastic properties (5). Changes in MUC5B structure with PAAG treatment translated to improved viscosity of purified MUC5B, and ultimately other more complex model systems, including primary cell culture and expectorated sputum samples. We have found previously that viscosity has a profound effect on the transportability of mucus in CF rats (34), with dynamic viscosities greater than 50-100 Pa•s resulting in a profound inhibition of airway transport. Data showing improvement in airway transport concurrent with improvement in viscosity (Figure 2J) substantiate this finding, and underscore the importance of viscosity as a therapeutic target in $\mathrm{CF}$, even in the absence of mucus hydration. PAAG also had effects on sputum elasticity (Figure 1B). Whether this activity of the molecule could be exploited for diseases in which elasticity is particularly problematic, such as status asthmaticus, deserves further exploration. 
The effect of PAAG on mucin was partially dependent on $\mathrm{Ca}^{2+}$ concentration, underscoring the concept that a bioactive biopolymer to affect electrostatic interactions represents a new avenue that can be exploited to interrupt pathogenesis of disease. PAAG has a unique physical mechanism that underlies its ability to interact with mucins and GAGs to accomplish these effects. First, PAAG is a high-MW polycationic polymer. Polycations by their nature are entropically constrained relative to free ions, as the individual components of the polymer cannot diffuse freely. The positively charged arginine moieties on the polyglucosamine backbone of PAAG favor displacement of $\mathrm{Ca}^{2+}$, which as a divalent cation functions to provide charge shielding and cohesion among mucins, or between mucins and cell surface GAGs in the respiratory (8) and GI tracts (43). This process is thermodynamically favored by freeing $\mathrm{Ca}^{2+}$ at the minimum expense of binding a large, already entropically constrained polymer $(44,45) . \mathrm{Ca}^{2+}$ also has a key role in altering domain binding at the N-terminus of MUC5B, which is crucial to its condensed (and more viscous) structure $(29,46)$. This is consistent with the nonpharmacologic use of polycationic polymers used to displace divalent or multivalent cations in many systems, such as at membrane interfaces; charge shielding serves to replace the role of bicarbonate-mediated $\mathrm{Ca}^{2+}$ chelation that is disordered in $\mathrm{CF}$ (47). Note that this does not rule out the role of other enzymatically driven reactions that may also function to make mucus more adherent or viscous, as shown in the GI tract (48).

Second, the positive charge and the specific type of cation are critical to the mechanism of PAAG (49). The effect of PAAG on mucin was partially dependent on $\mathrm{Ca}^{2+}$ solution concentration, with higher $\mathrm{Ca}^{2+}$ saturating the mucins and interfering with PAAG activity. Guanidinium hydrochloride is a ubiquitous chaotropic agent for protein and DNA denaturalization. The guanidinium group provided by arginine modifications provides a positive charge that, unlike $\mathrm{Ca}^{2+}$, serves as a chaotropic agent that solubilizes proteins and biopolymers, including purified mucins $(14,50)$. Consequently, we observe that when the $\mathrm{Ca}^{2+}$ is displaced, the mucins develop a more linear structure. Noting these mechanisms, PAAG has already exhibited safety by both inhaled and intravenous administration in an array of preclinical assessments, and appears suitable for human use in a study of healthy volunteers (our unpublished observations). While PAAG may also interact with extracellular DNA due to the negative charge of the phosphate backbone, we suspect mucin interactions are the dominant mechanism, based on relative concentrations of these molecules expected in the airway.

As a consequence of $\mathrm{Ca}^{2+}$ displacement, increased local concentrations may occur, resulting in mucin aggregation in those locations. These local $\mathrm{Ca}^{2+}$ concentrations may explain (i) how mucin forms are dichotomized into both beneficial linear, slowly migrating forms and compact clumps that migrate quickly (Supplemental Figures 5-7); and (ii) the propensity for patchy appearance of PAAG along the surface epithelium (Figure 1K and Supplemental Figure 3). Regardless, the effect of the linear, more transportable mucin forms seems to dominate the effects of PAAG on the viscoelastic properties of mucus. Other divalent cations are also found in mucus, but at a much lower concentration than $\mathrm{Ca}^{2+}$; however, their role in their contribution to mucus viscosity as compared with $\mathrm{Ca}^{2+}$ has been questioned, and PAAG would be expected to displace them by similar electrostatic mechanisms (51).

The use of a small glycopolymers to chelate $\mathrm{Ca}^{2+}$ as a means to favorably alter mucus viscoelasticity in $\mathrm{CF}$ lung disease has been investigated recently (10-12). While this is mechanistically related to the effects described here, the use of a high-MW glycopolymer that interacts directly with mucins and the glycocalyx to displace $\mathrm{Ca}^{2+}$ binding is a distinct approach. Large biopolymers produced from polysaccharides, proteins, and nucleic acids are currently used in tissue engineering and to aid in the delivery of therapeutic molecules such as drugs and genes, but their use as a pharmaceutical itself is, to our knowledge, a unique application (52). PAAG is synthetically modified to directly interact with anionic sulfates and carboxylates on glycosaminoglycans and mucins (53). In contrast, chelating agents such as OligoG, a short, naturally occurring glycopolymer, target $\mathrm{Ca}^{2+}$ and other divalent cations to reduce their binding to mucins or the glycocalyx. Although initial clinical trials with OligoG were not definitive in patients with CF (54), interacting with pathologic CF mucins directly via PAAG may have inherent advantages as compared with indirectly addressing this by depleting free $\mathrm{Ca}^{2+}$, since the concentrations of chelating agents required to induce such effects are high (13); this could conceivably precipitate adverse effects, disrupt epithelial integrity (55), and diminish $\mathrm{Cl}^{-}$secretion (56). Noting that biofilm extracellular biopolymer substances exhibit polyanionic structure and electrostatic properties similar to those of mucins (57), and low-WM Ca ${ }^{2+}$ chelators disrupt biofilms $(58,59)$, bacterial biofilms may also be a potential target for PAAG.

In summary, we demonstrate the potential use of a high-MW glycopolymer as a therapeutic agent. These findings demonstrate the potential of large, targeted biopolymers to treat $\mathrm{CF}$ and other diseases affected by abnormal mucus, opening new possibilities in clinical medicine across a broad disease spectrum. 


\section{Methods}

Procurement and preparation of human sputum. Sputum specimens were obtained from patients with CF hospitalized for pulmonary exacerbation. After collection, the specimen was kept on ice until tested. Sputum was then divided into 200- $\mu$ l aliquots, treated with polyacetyl arginyl glucosamine (PAAG, specifically PAAG15A from Synedgen Inc.) at a concentration of 100 or $500 \mu \mathrm{g} / \mathrm{ml}, 100 \mu \mathrm{g} / \mathrm{ml} \mathrm{HPMC}$, PBS, DNase (Sigma-Aldrich); $100 \mu \mathrm{g} / \mathrm{ml}$ (DNase), $10 \mathrm{mM} \mathrm{EGTA} \mathrm{(Sigma-Aldrich),} \mathrm{or} 100 \mu \mathrm{g} / \mathrm{ml}$ DTT (Sigma-Aldrich); and placed in a $37^{\circ} \mathrm{C}$ water bath for 2 hours. Sputum was then transferred to cone and plate rheometer for measurement of viscoelastic properties.

Measurement of viscosity and elasticity. Shear-dependent viscosity was measured with a TA Instruments Discovery Series HRII Rheometer. After a 10-minute conditioning period, a flow ramp procedure began, with initial stress $1.0 \times 10^{-3}$ to $10 \mathrm{~Pa}$ for 600 seconds in log mode. Five points were observed per decade, and oversampling of controlled stress was checked. An oscillation frequency procedure immediately followed with $5 \%$ strain, a logarithmic sweep from 0.05 to $20 \mathrm{~Hz}, 10$ points observed per decade, continuous direct controlled strain, and 3-second conditioning time with 3-second sampling time data acquisition parameters. The elasticity values were recorded from the oscillation frequency procedure. A flow sweep procedure then made a logarithmic sweep from 0.02 to $1000 \mathrm{~s}^{-1}$, and 5 points per decade were recorded, followed by a 5 -second equilibration time and a 10 -second averaging time. The motor mode for controlled rate was set to automatic. The viscosity values were recorded from the flow sweep procedure (23).

Percent solid content measurements. Expectorated CF sputum was treated as described above during bulk rheology measurements. $5 \mu \mathrm{l}$ of treated sputum was weighed to determine wet weight. Samples were left overnight in a dry oven for liquid evaporation, and weighed again for dry weight. All weights were taken using a UMX2 Ultra-microbalance (Mettler Toledo). Wet and dry weights were used to calculate the fraction that was solid content.

Procurement and growth of epithelial cells. Primary HBE cells were derived from lung explants from either normal patients or patients with cystic fibrosis. In brief, first- or second-passage cells, which underwent expansion and attained confluency, were seeded onto 6.5 -mm-diameter permeable supports $\left(0.5 \times 10^{6}\right.$ cells per filter; Corning Inc.) coated with NIH 3T3 fibroblast conditioned media. Cells were grown in media to induce terminal differentiation for at least 6-8 weeks $(20,24)$.

Fluorescence microscopy. HBE cells obtained from CF patients homozygous for Phe508del were washed in PBS for 15 minutes, treated with various concentrations of PAAG, and combined with $10 \mu$ of 500 -nm fluorescent poly(ethylene glycol) polystyrene beads (PEG_PS) in a 1:120 dilution in PBS as shown in previously (23). The MCT of the cells was then observed by $\mu \mathrm{OCT}$ imaging techniques. The cells were then treated with benzalkonium chloride $0.01 \%$ media mixture for an hour to stop ciliary motion. The cells were washed in media for 15 minutes and then allowed to incubate at $37^{\circ} \mathrm{C}$ for at least 3 hours. A fluorescence microscope and MetaMorph software were used to take videos of the fluorescent particles in the ASL layer of the cells. Fluorescence time-series images were acquired using an inverted microscope at a frame rate of approximately 17 frames/ second. Four videos of different regions of interest in different quadrants in the filter were recorded (30).

PTM. ImageJ (NIH) software was then used to track the Brownian motion of the fluorescent nanoparticles in mucus. One hundred particles were tracked per well ( 25 per region of interest), and the coordinates obtained were entered into a MATLAB script that generated MSD value for each particle. Bulk movement was removed by the tracking algorithm (23).

$\mu O C T$ imaging. Measurements of functional microanatomic parameters in cultured cells and tissues were performed using $\mu \mathrm{OCT}$, a high-speed, high-resolution microscopic reflectance imaging modality (28). The $\mu \mathrm{OCT}$ instrument provides cross-sectional images of the epithelium with a resolution sufficient to directly visualize and quantify micro-anatomic parameters including ASL depth, periciliary liquid (PCL) depth, ciliary beat frequency $(\mathrm{CBF})$, and $\mathrm{MCT}$ rates without using exogenous dyes or particles. Images are acquired at a rate of 40 frames per second and at 512 lines per frame. Quantitative analysis of ASL and PCL depths were characterized directly by geometric measurement of the respective layers, and images over several frames captured the length of fully extended cilia. MCT rate was determined using time elapsed and distance traveled of native particulates in the mucus over multiple frames. For each HBE monolayer, images were acquired $1 \mathrm{~mm}$ from the filter periphery with a scanning beam parallel to the tangent of the circumference of the filter membrane disc. For rat trachea, the imaging beam was placed at several locations along the ventral surface of the trachea $(20,24)$. MATLAB code to derive quantitative parameters from $\mu$ OCT images was written by the authors and is housed at $\mathrm{UAB}$ and $\mathrm{MGH}$. The code is available on request. 
In situ localization of PAAG. Phe508del HBE monolayers were treated with $10 \mu \mathrm{l}$ of $250 \mu \mathrm{l} / \mathrm{ml}$ FITC-labeled PAAG (PAAG15A-FITC, Synedgen Inc.). For contrast, cells were stained with $600 \mu$ CellTracker Violet BMQC (Invitrogen) $(15 \mu \mathrm{M})$ at $37^{\circ} \mathrm{C}$ for 45 minutes, and the ASL was stained with $25-30 \mu 1$ of 10 $\mathrm{mg} / \mathrm{ml}$ Dextran, Texas Red (Invitrogen)/FC-70 (Acros Organics) to prevent evaporation, then incubated at room temperature for 15 minutes. Transwell membranes were placed in a sterile glass-bottom dish (MatTek) containing $150 \mu \mathrm{PBS}$. Images were acquired using an LSM710 Carl Zeiss confocal laser scanning microscope with a $\times 20$ air objective and $Z$-stack scanning (30).

In vivo localization of PAAG. FITC-PAAG $(1.5 \mathrm{ml}$ of $200 \mu \mathrm{g} / \mathrm{ml}$ in $1.38 \%$ glycerol) or vehicle was nebulized to WT ferrets using an over-the-counter nebulizer $(3.51 / \mathrm{min}, 14.5 \mathrm{PSI})$ over 15 minutes in a standard cage. After completion, 15 minutes were allowed before the animal was sacrificed with intraperitoneal Euthasol. Lungs were dissected, embedded in optimal cutting temperature (O.C.T.) medium and rapidly frozen in 2-methylbutane and crushed dry ice. Tissue was sectioned at $8 \mu \mathrm{m}$ onto slides and fixed in 4\% paraformaldehyde for 20 minutes. Nuclei were stained with Hoechst 33342 at 1:1000. Confocal images were obtained on a Zeiss LSM 700 laser scanning microscope at $\times 20$ using identical settings, and images were minimally processed following capture.

Effect of PAAG on sputum transport ex vivo. To assess the effect of PAAG on sputum transportability, $\mathrm{CF}$ sputum was collected and stored at $4^{\circ} \mathrm{C}$ overnight. $200 \mu 1$ sputum was treated with $20 \mu 1 \mathrm{PBS}$ control, $100 \mu \mathrm{g} / \mathrm{ml}$ PAAG, $250 \mu \mathrm{g} / \mathrm{ml}$ PAAG, or $250 \mu \mathrm{g} / \mathrm{ml}$ DTT and placed in a $37^{\circ} \mathrm{C}$ water bath for 2 hours. Trachea was removed from adult non-CF rat and washed twice with $500 \mu 1$ PBS before baseline imaging using $\mu$ OCT. $3 \mu \mathrm{l}$ of each sputum sample was added to the distal end of the trachea, and $\mu \mathrm{OCT}$ imaging was taken distal to that location. This was repeated at 3 anatomic locations for each sample addition; at least 3 measures were obtained from each region of interest. Tracheas were washed twice with $500 \mu 1$ PBS between each sample. The order of sample additions was randomized. Images with PBS were obtained at the end of the experiment to confirm viability of the trachea.

Expression of N-terminal construct of MUC5B and analytical ultracentrifugation. The $\mathrm{N}$-terminal construct of MUC5B (NT5B [26-1303 aa]: UniProtKB accession number Q9HC84) was expressed in mammalian 293-EBNA cells, as previously described (29). Briefly, conditioned media was purified using nickel affinity chromatography, then dimer-enriched fractions were collected following size exclusion chromatography using a Superose 6 10/300GL column (GE Healthcare), prior to ion exchange chromatography.

For analytical ultracentrifugation (AUC), samples in HBS (25 mM HEPES, $200 \mathrm{mM} \mathrm{NaCl}$ ) were incubated with $10 \mathrm{mM} \mathrm{CaCl}_{2}$ at $\mathrm{pH} 5.5$ at $4^{\circ} \mathrm{C}$, with rotation overnight, to form $\mathrm{N}$-terminal multimers. The sample was split into 3 aliquots; one aliquot was retained for analysis, $100 \mu \mathrm{g} / \mathrm{ml}$ PAAG was added to the second aliquot, and $100 \mu \mathrm{g} / \mathrm{ml} \mathrm{HPMC}$ was added to the third aliquot ( $\mathrm{pH}$ was maintained at 5.5). All samples were incubated overnight at $4^{\circ} \mathrm{C}$ with rotation. Samples were analyzed using velocity experiments on an Optima XL-A ultracentrifuge (Beckman Instruments), centrifuging in a double sector cell at $93,980 \mathrm{~g}$, with 200 scans taken at 1.5 -minute intervals at $280 \mathrm{~nm}$, at $20^{\circ} \mathrm{C}$. The sedimentation coefficients were determined using SedFit version $13.0 \mathrm{~b}(60)$.

MUC5B preparation. Purified MUC5B samples were diluted in a $0.1-\mathrm{M} \mathrm{NaCl} / 0.01-\mathrm{M}$ Tris- $\mathrm{HCl}$ buffer, producing a final mucin concentration of $50 \mu \mathrm{g} / \mathrm{ml}$ for electron microscopy and $150 \mu \mathrm{g} / \mathrm{ml}$ for rate-zonal analysis, PTM, and size exclusion chromatography with multi-angle laser light scattering (SEC-MALS). Mucins were then incubated for 24 hours with either $10 \mu \mathrm{g} / \mathrm{ml} \mathrm{Ca}{ }^{2+}$ or an equivalent volume of EM buffer. Samples were then incubated overnight with varying concentrations of PAAG $(25,100,250 \mu \mathrm{g} / \mathrm{ml})$ or $100 \mu \mathrm{g} / \mathrm{ml} \mathrm{HPMC}$.

Transmission electron microscopy. Samples were applied onto a glow discharged carbon coated 400 mesh copper grid (Electron Microscopy Sciences) and incubated for 30 seconds. Grids were then washed in $\mathrm{ddH} 2 \mathrm{O}$ and subsequently negatively stained with $2 \%(\mathrm{w} / \mathrm{v})$ uranyl acetate for 1 minute. Using a Tecnai BioTwin at $100 \mathrm{Kv}$, transmission electron microscopy (TEM) data were recorded. Micrographs were then acquired using a Gatan Orius CCD camera.

Rate-zonal centrifugation. Rate-zonal centrifugations were performed as a measure of mucin mass and shape. Mucins were centrifuged at $210,000 \mathrm{~g}$ for 1 hour 15 minutes at $15^{\circ} \mathrm{C}$ using $10 \%-35 \%(\mathrm{w} / \mathrm{v})$ sucrose gradients. Samples were layered onto preformed gradients and centrifuged in a Beckman SW40 Ti centrifuge using the parameters stated above. After centrifugation, tubes were fractionated from the top of the gradient and 24 fractions collected. The sucrose concentration of each fraction was then determined through measuring refractive index. Fractions were transferred onto nitrocellulose membranes by slot blotting, and MUC5B was subsequently detected by periodic acid-Schiff staining 
(PAS) or immunostaining with the monoclonal antibody EUMUC5B $(61,62)$. PAS staining intensity was analyzed using ImageJ and EUMUC5B staining (used at a dilution of 1:2000) was analyzed using an Odyssey Infrared Imaging System (LI-COR).

SEC-MALS. SEC-MALS was performed to allow for the determination of mucin concentration and molecular weight. Samples were applied onto a Superose 6 10/300 column (GE Healthcare) and eluted in running buffer at a flow rate of $0.31 \mathrm{ml} / \mathrm{min}$. Eluents were passed through an inline DAWN EOS laser photometer (Wyatt) followed by an Optilab rEX refractometer with quasielastic light scattering dynamic light scattering attachment (Wyatt). Using a dn/dc value of $0.165 \mathrm{ml} / \mathrm{g}$, light scattering (LS) and refractive index (RI) measurements were then taken and analyzed with ASTRA 6 software $(29,60)$.

Intestinal studies in CFTR-knockout mouse model. The generation of congenic C57BL/6J.Cftrtm1Unc/J (CF) mice has been described previously (33). WT and CF mice of both sexes at weaning age (4-5 weeks) were randomized and administered PAAG $(40 \mathrm{mg} / \mathrm{kg} / \mathrm{d}$ ) by oral gavage divided 3 times daily (or vehicle control) for 21 days while on a regular diet. Body weights and general health were monitored daily. Sample sizes were based on known mortality rates in CF mice, and all animals that entered the study were included in the final analysis, except a single cohort found to be infected with mites, which required concurrent treatment with ivermectin and interacted with PAAG treatment (33). Following euthanasia for clinical deterioration or at the end of the treatment period, intestines were collected for gross pathology and histopathologic analysis. Mice were euthanized on day 21 of treatment, and whole intestinal tract was imaged grossly, fixed using 70\% alcoholic formalin, and embedded in paraffin. Slides were stained with $\mathrm{H} \& \mathrm{E}$ and $\mathrm{AB} / \mathrm{PAS}$ to evaluate mucus impaction in the intestine. Animals were randomized into treatment groups, but in order to administer treatments, the researcher could not be blinded to the protocol.

En bloc MCT in CFTR-knockout rat model. The airway mucus transport defect of $C f r^{-1}$ rats has been described previously (34). Rats were aged to 6 months. Upon euthanasia, trachea and lung were excised and opened along the dorsal surface from the larynx to bronchi. They were then placed onto an F12 media-soaked gauze and allowed to equilibrate to physiologic conditions $\left(37^{\circ} \mathrm{C}, 5 \% \mathrm{CO}_{2}, 100 \%\right.$ humidity) for 30 minutes before imaging. We have previously described $\mu \mathrm{OCT}$ methods for interrogation of airway epithelia and quantitative image analysis (28). In brief, MCT rate was determined using time elapsed and distance traveled of native particulates in the mucus over multiple frames. Standard distances along each trachea were used.

CFTR-knockout ferret model. The generation of CFTR-knockout (CF) ferrets has been described previously $(35,36)$. Heterozygous $\mathrm{KO}$ founders were crossed, and progeny were studied. CF ferrets were reared on combination antibiotics, all dosed twice daily: piperacillin/tazobactam ( $4 \mathrm{mg} / \mathrm{kg}$; Wyeth Pharmaceuticals Inc.), enrofloxacin (10 mg/kg; Bayer Healthcare LLC), and metronidazole (20 mg/kg; Hospira Inc.). Each injection was administered in $100 \mu 1$ saline subcutaneously. CF ferrets were housed individually, and careful attention was paid to bowel function, with PEG3350 titrated to routine body weights and fecal morphology. No sample size estimates were conducted in ferrets since to our knowledge no drug by this mechanism had yet been assessed in ferrets; all ferrets treated in the study were included in the final analysis.

CT imaging. The CF ferret was lightly anesthetized with subcutaneous ketamine $(5-20 \mathrm{mg} / \mathrm{kg})$ and xylazine $(0.25-5 \mathrm{mg} / \mathrm{kg})$, then placed in a standardized position on the CT gantry. High-resolution CT images were acquired with a Siemens Somatom Force scanner $(2 \times 192$ slices $)$ and viewed on OsiriX/ Horos DICOM software, where a similar plane was identified and captured across all time intervals.

Bronchoscopy. Ferrets underwent flexible fiberoptic bronchoscopy on the indicated days following anesthesia with ketamine/xylazine, as detailed above. Sedation was maintained with isoflurane $(0 \%-5 \%)$ in supplemental oxygen. During the procedure, vitals (pulse, respiratory rate, oxygen saturation, and temperature) were monitored. The bronchoscope (Pentax FB-8V) was inserted orally through the larynx with the aid of a standard pediatric laryngoscope (Miller blade, size 0 ). The suction channel was not used until the bronchoscope tip was wedged into the desired airway. Two to 3 aliquots $(1-1.5 \mathrm{ml} / \mathrm{kg}$ body weight each; total of $3 \mathrm{ml} / \mathrm{kg}$ ) of sterile $0.9 \%$ saline were instilled and then recovered with gentle aspiration. When PAAG was used, 2 aliquots of $1.5 \mathrm{ml}(500 \mu \mathrm{g} / \mathrm{ml}$, total dose $1500 \mu \mathrm{g})$ were instilled in the indicated lung segment, left to dwell for 1-5 minutes, and then aspirated by gentle aspiration. For aerosolized PAAG delivery, an over-the-counter ambulatory nebulizer (3.5 1/min, $14.5 \mathrm{PSI})$ was used to administer $1.5 \mathrm{ml}$ PAAG $(250 \mu \mathrm{g} / \mathrm{ml}$, total dose $375 \mu \mathrm{g})$ over 15 minutes in a standard cage twice weekly.

Proteomics analysis of ferret BAL samples treated with PAAG. PAAG $(1.5 \mathrm{ml}$ of $200 \mu \mathrm{g} / \mathrm{ml} \mathrm{in} 1.38 \%$ glycerol) or vehicle was nebulized to $C f t r^{-1}$ ferrets using an over-the-counter nebulizer (3.5 1/min, $\left.14.5 \mathrm{PSI}\right)$ over 15 minutes in a standard cage twice weekly for 8 weeks. Proteomics of ferret BAL samples were per- 
formed as previously described (63) prior to and after PAAG exposure (43). Briefly, $10 \mu \mathrm{g}$ protein BAL protein was reduced, alkylated, and then digested with trypsin overnight. Paired samples from each animal (untreated and PAAG treated) were then dimethyl labeled with a "light" or "heavy" stable isotope. Untreated samples were treated with $4 \mu \mathrm{l}$ of $4 \%$ light formaldehyde $\left(\mathrm{CH}_{2} \mathrm{O}\right)$, and PAAG-treated samples were treated with $4 \mu \mathrm{l}$ heavy formaldehyde $\left({ }^{13} \mathrm{CCD}_{2} \mathrm{O}\right)$. Labeled untreated and PAAG pairs were then mixed and desalted using a $\mathrm{C}_{18}$-MicroSpin column (The Nest Group Inc.). Peptides were then evaluated on an Orbitrap Fusion Lumos mass spectrometer (Thermo Fisher Scientific).

Reagents and quality assurance. PAAG (PAAG15A, being developed as SNSP113 by Synspiria Inc.) was provided by Synedgen Inc. PAAG used here is near GMP-grade material almost identical to the GMPgrade drug substance that has been advanced to clinical testing. The MW of PAAG polymers found in the drug substance have a distribution from 50 to $150 \mathrm{kDa}$. HPMC was also provided by Synedgen Inc. All other agents were purchased from commercial providers, as noted. CF and non-CF cell lines were routinely tested for mycoplasma contamination on an annual basis.

Statistics. Statistical analysis was performed in GraphPad Prism version 6.0 or greater. Comparisons were made using unpaired $t$ test or ANOVA with Kruskal-Wallis test with Dunn's MCT (for log-transformed data). The data on expectorated sputum in Figure 1, C-D, were log transformed and then analyzed using paired $t$ test, since data were not normally distributed. Nonparametric statistical tests were used for $\mathrm{MCT}$, as rates were not normally distributed, as previously described. Sample sizes for in vitro studies were based on prior experience with microrheology (2) or MCT (24). All statistical tests were 2-sided, and $P$ values less than 0.05 were considered significant. Variances between groups were equal in all cases, unless otherwise noted, as assessed by equal variance testing prior to inferential statistics. Statistics are presented as mean \pm SEM unless indicated otherwise.

Study approval. Use of animals and tissues was approved by UAB and University of Iowa IACUCs. Use of primary HBE cells and sputum samples was approved by the IRB of UAB and Cincinnati Children's Hospital. Written informed consent was received from all participants who provided sputum samples and for acquisition of airway tissues to procure primary human airway cells.

\section{Author contributions}

CMFP, GWH, BHR, SMT, SEB, BAG, JFE, DJT, WES, WPW, SMB, and SMR conceived of the experiments; CMFP, GWH, BHR, HLB, JDW, SMT, CS, CER, KKC, SEB, YL, HML, MM, BAG, TIAE, and HH conducted research; CMFP, GWH, BHR, HLB, JDW, SMT, CS, CER, KKC, SEB, YL, HML, EFL, BAG, JH, GJT, JPC, JFE, DJT, SMB, and SMR analyzed the data; CMFP, JDW, EFL, DJT, SMB and SMR wrote the manuscript; SMB and SMR supervised the project.

\section{Acknowledgments}

The authors thank the patients with CF who volunteered for these studies. The authors acknowledge assistance from Tambra Roberts, Katie Brand, Scott House, and Justin Wade for obtaining CF sputum from volunteers and Kathryn Duris for images of FITC-PAAG. The authors acknowledge a number of helpful discussion with members of the CF Foundation's Mucus Clearance Consortium. This research was sponsored by the NIH, including R43HL118867 (to Synedgen Inc.), R01HL1116213, R35HL135816, and P30DK072482 (to SMR), R01HL125169 (to JH), and R24 HL123482 and P30 DK054759 (to JFE); and by the Cystic Fibrosis Foundation, including the Mucociliary Clearance Consortium (ROWE10XX0 to SMR, THORNT07XXX0 to DJT, and HANES07XX0 and HANES16XX0 to JH), ROSEN17XX0 (to BHR), and the Research Development Program (R464-CF11 to the UAB CF Research Center). The Wellcome Trust Centre for Cell-Matrix Research, University of Manchester, is supported by core funding from the Wellcome Trust (grant 088785/Z/09/Z). CMF is currently supported by NIH-NIGMS T32GM008111. GWH is supported by a studentship from the Medical Research Council (United Kingdom).

Address correspondence to: Steven M. Rowe, MCLM 7061918 University Blvd, Birmingham, Alabama 35294-0006, USA. Phone: 205.934.9640; Email: smrowe@uab.edu.

1. Ratjen F, Bell SC, Rowe SM, Goss CH, Quittner AL, Bush A. Cystic fibrosis. Nat Rev Dis Primers. 2015;1:15010.

2. Lai SK, Wang YY, Wirtz D, Hanes J. Micro- and macrorheology of mucus. Adv Drug Deliv Rev. 2009;61(2):86-100.

3. Costerton JW, et al. Bacterial biofilms in nature and disease. Annu Rev Microbiol. 1987;41:435-464. 
4. Fahy JV, Dickey BF. Airway mucus function and dysfunction. N Engl J Med. 2010;363(23):2233-2247.

5. Quinton PM. Cystic fibrosis: impaired bicarbonate secretion and mucoviscidosis. Lancet. 2008;372(9636):415-417.

6. Garcia MA, Yang N, Quinton PM. Normal mouse intestinal mucus release requires cystic fibrosis transmembrane regulator-dependent bicarbonate secretion. J Clin Invest. 2009;119(9):2613-2622.

7. Gustafsson JK, et al. Bicarbonate and functional CFTR channel are required for proper mucin secretion and link cystic fibrosis with its mucus phenotype. J Exp Med. 2012;209(7):1263-1272.

8. Kesimer M, Makhov AM, Griffith JD, Verdugo P, Sheehan JK. Unpacking a gel-forming mucin: a view of MUC5B organization after granular release. Am J Physiol Lung Cell Mol Physiol. 2010;298(1):L15-22.

9. Hoegger MJ, et al. Cystic fibrosis. Impaired mucus detachment disrupts mucociliary transport in a piglet model of cystic fibrosis. Science. 2014;345(6198):818-822.

10. Pritchard MF, et al. A new class of safe oligosaccharide polymer therapy to modify the mucus barrier of chronic respiratory disease. Mol Pharm. 2016;13(3):863-872.

11. Vitko M, et al. A novel guluronate oligomer improves intestinal transit and survival in cystic fibrosis mice. J Cyst Fibros. 2016;15(6):745-751

12. Ermund A, et al. OligoG CF-5/20 normalizes cystic fibrosis mucus by chelating calcium. Clin Exp Pharmacol Physiol. 2017;44(6):639-647

13. Ermund A, Meiss LN, Gustafsson JK, Hansson GC. Hyper-osmolarity and calcium chelation: effects on cystic fibrosis mucus. Eur J Pharmacol. 2015;764:109-117.

14. Schneider CP, Shukla D, Trout BL. Arginine and the Hofmeister series: the role of ion-ion interactions in protein aggregation suppression. J Phys Chem B. 2011;115(22):7447-7458.

15. Dawson M, Wirtz D, Hanes J. Enhanced viscoelasticity of human cystic fibrotic sputum correlates with increasing microheterogeneity in particle transport. J Biol Chem. 2003;278(50):50393-50401.

16. Matsui H, Randell SH, Peretti SW, Davis CW, Boucher RC. Coordinated clearance of periciliary liquid and mucus from airway surfaces. J Clin Invest. 1998;102(6):1125-1131.

17. Shak S, Capon DJ, Hellmiss R, Marsters SA, Baker CL. Recombinant human DNase I reduces the viscosity of cystic fibrosis sputum. Proc Natl Acad Sci US A. 1990;87(23):9188-9192.

18. Souza-Fernandes AB, Pelosi P, Rocco PR. Bench-to-bedside review: the role of glycosaminoglycans in respiratory disease. Crit Care. 2006;10(6):237.

19. Matsui H, et al. Evidence for periciliary liquid layer depletion, not abnormal ion composition, in the pathogenesis of cystic fibrosis airways disease. Cell. 1998;95(7):1005-1015.

20. Birket SE, et al. Combination therapy with cystic fibrosis transmembrane conductance regulator modulators augment the airway functional microanatomy. Am J Physiol Lung Cell Mol Physiol. 2016;310(10):L928-L939.

21. Neuberger T, Burton B, Clark H, Van Goor F. Use of primary cultures of human bronchial epithelial cells isolated from cystic fibrosis patients for the pre-clinical testing of CFTR modulators. Methods Mol Biol. 2011;741:39-54.

22. Van Goor F, et al. Rescue of CF airway epithelial cell function in vitro by a CFTR potentiator, VX-770. Proc Natl Acad Sci U S A 2009;106(44):18825-18830.

23. Chu KK, et al. Particle-tracking microrheology using micro-optical coherence tomography. Biophys J. 2016;111(5):1053-1063.

24. Birket SE, et al. A functional anatomic defect of the cystic fibrosis airway. Am J Respir Crit Care Med. 2014;190(4):421-432.

25. Suh J, Dawson M, Hanes J. Real-time multiple-particle tracking: applications to drug and gene delivery. Adv Drug Deliv Rev. 2005;57(1):63-78.

26. Liu J, Walker NM, Ootani A, Strubberg AM, Clarke LL. Defective goblet cell exocytosis contributes to murine cystic fibrosis-associated intestinal disease. J Clin Invest. 2015;125(3):1056-1068.

27. Ambort D, et al. Calcium and $\mathrm{pH}$-dependent packing and release of the gel-forming MUC2 mucin. Proc Natl Acad Sci U S A. 2012;109(15):5645-5650.

28. Liu L, et al. Method for quantitative study of airway functional microanatomy using micro-optical coherence tomography. PLoS One. 2013;8(1):e54473.

29. Ridley C, et al. Assembly of the respiratory mucin MUC5B: a new model for a gel-forming mucin. J Biol Chem. 2014;289(23):16409-16420.

30. Raju SV, et al. Cigarette smoke induces systemic defects in cystic fibrosis transmembrane conductance regulator function. Am J Respir Crit Care Med. 2013;188(11):1321-1330.

31. Kesimer M, Ehre C, Burns KA, Davis CW, Sheehan JK, Pickles RJ. Molecular organization of the mucins and glycocalyx underlying mucus transport over mucosal surfaces of the airways. Mucosal Immunol. 2013;6(2):379-392.

32. Yuan S, et al. Oxidation increases mucin polymer cross-links to stiffen airway mucus gels. Sci Transl Med. 2015;7(276):276ra27.

33. Snouwaert JN, et al. An animal model for cystic fibrosis made by gene targeting. Science. 1992;257(5073):1083-1088.

34. Birket SE, et al. Development of an airway mucus defect in the cystic fibrosis rat. JCI Insight. 2018;3(1):e97199.

35. Sun X, et al. Lung phenotype of juvenile and adult cystic fibrosis transmembrane conductance regulator-knockout ferrets. Am $J$ Respir Cell Mol Biol. 2014;50(3):502-512.

36. Sun X, et al. Disease phenotype of a ferret CFTR-knockout model of cystic fibrosis. J Clin Invest. 2010;120(9):3149-3160.

37. Shah VS, et al. Airway acidification initiates host defense abnormalities in cystic fibrosis mice. Science. 2016;351(6272):503-507.

38. Donaldson SH, Bennett WD, Zeman KL, Knowles MR, Tarran R, Boucher RC. Mucus clearance and lung function in cystic fibrosis with hypertonic saline. N Engl J Med. 2006;354(3):241-250.

39. Elkins MR, et al. A controlled trial of long-term inhaled hypertonic saline in patients with cystic fibrosis. $N E n g l J M e d$. 2006;354(3):229-240.

40. Trillo-Muyo S, et al. Granule-stored MUC5B mucins are packed by the non-covalent formation of N-terminal head-to-head tetramers. J Biol Chem. 2018;293(15):5746-5754.

41. Ermund A, Meiss LN, Dolan B, Bahr A, Klymiuk N, Hansson GC. The mucus bundles responsible for airway cleaning are retained in cystic fibrosis and by cholinergic stimulation. Eur Respir J. 2018;52(2):1800457.

42. Ermund A, et al. The normal trachea is cleaned by MUC5B mucin bundles from the submucosal glands coated with the 
MUC5AC mucin. Biochem Biophys Res Commun. 2017;492(3):331-337.

43. Birchenough GM, Johansson ME, Gustafsson JK, Bergstrom JH, Hansson GC. New developments in goblet cell mucus secretion and function. Mucosal immunology. 2015;8(4):712-719.

44. de Kruif CG, Weinbreck F, de Vries R. Complex coacervation of proteins and anionic polysaccharides. Curr Opin Colloid Interface Sci. 2004;9(5):340-349.

45. Ou Z, Muthukumar M. Entropy and enthalpy of polyelectrolyte complexation: Langevin dynamics simulations. J Chem Phys. 2006;124(15):154902.

46. Ridley C, Kirkham S, Williamson SJ, Davis CW, Woodman P, Thornton DJ. Biosynthesis of the polymeric gel-forming mucin MUC5B. Am J Physiol Lung Cell Mol Physiol. 2016;310(10):L993-L1002.

47. Vaara M. Agents that increase the permeability of the outer membrane. Microbiological Reviews. 1992;56(3):395-411.

48. Schutte A, et al. Microbial-induced meprin beta cleavage in MUC2 mucin and a functional CFTR channel are required to release anchored small intestinal mucus. Proc Natl Acad Sci U S A. 2014;111(34):12396-12401.

49. Collins KD. Why continuum electrostatics theories cannot explain biological structure, polyelectrolytes or ionic strength effects in ion-protein interactions. Biophys Chem. 2012;167:43-59.

50. Sawyer WH, Puckridge J. The dissociation of proteins by chaotropic salts. J Biol Chem. 1973;248(24):8429-8433.

51. Tang XX, et al. Acidic pH increases airway surface liquid viscosity in cystic fibrosis. J Clin Invest. 2016;126(3):879-891.

52. Nitta SK, Numata K. Biopolymer-based nanoparticles for drug/gene delivery and tissue engineering. Int J Mol Sci. 2013;14(1):1629-1654

53. Sterling JD, Baker SM. A continuum model of mucosa with glycan-ion pairing. Macromol Theory Simul. 2018;27(2):1700079.

54. Davies J, SMR2984 Study team. Oligo G: A Novel Mucolytic from Basic Science to Clinical Trials. Peds Pulm Supp. 2017;52(S47):150-151.

55. Vermeer PD, Panko L, Welsh MJ, Zabner J. erbB1 functions as a sensor of airway epithelial integrity by regulation of protein phosphatase 2A activity. J Biol Chem. 2006;281(3):1725-1730.

56. Middleton PG, Pollard KA, Donohoo E, Wheatley JR, Geddes DM, Alton EW. Airway surface liquid calcium modulates chloride permeability in the cystic fibrosis airway. Am J Respir Crit Care Med. 2003;168(10):1223-1226.

57. Flemming HC, Wingender J. The biofilm matrix. Nat Rev Microbiol. 2010;8(9):623-633.

58. Hengzhuang W, Song Z, Ciofu O, Onsøyen E, Rye PD, Høiby N. OligoG CF-5/20 Disruption of mucoid pseudomonas aeruginosa biofilm in a murine lung infection model. Antimicrob Agents Chemother. 2016;60(5):2620-2626.

59. Pritchard MF, et al. A low-molecular-weight alginate oligosaccharide disrupts pseudomonal microcolony formation and enhances antibiotic effectiveness. Antimicrob Agents Chemother. 2017;61(9):e00762-17.

60. Schuck P. Size-distribution analysis of macromolecules by sedimentation velocity ultracentrifugation and lamm equation modeling. Biophys J. 2000;78(3):1606-1619.

61. Rousseau K, Wickstrom C, Whitehouse DB, Carlstedt I, Swallow DM. New monoclonal antibodies to non-glycosylated domains of the secreted mucins MUC5B and MUC7. Hybrid Hybridomics. 2003;22(5):293-299.

62. Thornton DJ, Carlstedt I, Sheehan JK. Identification of glycoproteins on nitrocellulose membranes and gels. Mol Biotechnol. 1996;5(2):171-176.

63. Rosen BH, et al. Infection Is Not Required for Mucoinflammatory Lung Disease in CFTR-Knockout Ferrets. Am J Respir Crit Care Med. 2018;197(10):1308-1318. 